\title{
Glass-forming property of hydroxyectoine is the cause of its superior function as a desiccation protectant
}

\author{
Christoph Tanne ${ }^{1}$, Elena A. Golovina ${ }^{2}$, Folkert A. Hoekstra ${ }^{2}$, Andrea Meffert ${ }^{1}$ and Erwin A. Galinski ${ }^{1}$ * \\ ${ }^{1}$ Institute of Microbiology and Biotechnology, Rheinische Friedrich-Wilhelms-University Bonn, Bonn, Germany \\ ${ }^{2}$ Laboratory of Plant Physiology, Wageningen University, Wageningen, Netherlands
}

\author{
Edited by: \\ Aharon Oren, The Hebrew University \\ of Jerusalem, Israel \\ Reviewed by: \\ R. Thane Papke, University of \\ Connecticut, USA \\ Aharon Oren, The Hebrew University \\ of Jerusalem, Israel \\ ${ }^{*}$ Correspondence: \\ Erwin A. Galinski, Institute of \\ Microbiology and Biotechnology, \\ Rheinische Friedrich-Wilhelms- \\ University Bonn, Meckenheimer Allee \\ 168, Bonn 53115, Germany \\ e-mail:galinski@uni-bonn.de
}

We were able to demonstrate that hydroxyectoine, in contrast to ectoine, is a good glassforming compound. Fourier transform infrared and spin label electron spin resonance studies of dry ectoine and hydroxyectoine have shown that the superior glass-forming properties of hydroxyectoine result from stronger intermolecular $\mathrm{H}$-bonds with the $\mathrm{OH}$ group of hydroxyectoine. Spin probe experiments have also shown that better molecular immobilization in dry hydroxyectoine provides better redox stability of the molecules embedded in this dry matrix. With a glass transition temperature of $87^{\circ} \mathrm{C}$ (vs. $47^{\circ} \mathrm{C}$ for ectoine) hydroxyectoine displays remarkable desiccation protection properties, on a par with sucrose and trehalose. This explains its accumulation in response to increased salinity and elevated temperature by halophiles such as Halomonas elongata and its successful application in "anhydrobiotic engineering" of both enzymes and whole cells.

\footnotetext{
Keywords: hydroxyectoine, desiccation, glass transition temperature, enzyme stabilization, ESR, FTIR
}

\section{INTRODUCTION}

Compatible solutes (organic osmolytes) are low-molecular mass water-binding organic solutes, which are accumulated in the cytoplasm of halophiles for osmotic equilibrium, either as a replacement for or in combination with inorganic salts. They are also known as versatile stress-protecting compounds, in particular for the stabilization of proteins, membranes and whole cells. The cyclic amino acid derivative ectoine (Figure 1A) is one of the most common compatible solutes among halophilic heterotrophic Bacteria and has found diverse applications, above all as ingredient of skin care products (Graf et al., 2008). Organisms able to synthesize ectoine are often also able to convert this compound into $S, S$-1,4,5,6-tetrahydro-2-methyl-5-hydroxypyrimidine-4-carboxylate, hydroxyectoine (Figure 1B), by means of a 2-oxoglutarate dependent non-heme-iron(II) containing dioxygenase (Bursy et al., 2007; Reuter et al., 2010; Widderich et al., 2014). In Chromohalobacter salexigens, a member of the Halomonadaceae, the relative proportion of the hydroxylated derivative increases with salinity and/or temperature (Garcia-Estepa et al., 2006; Vargas et al., 2008). It has long been known that hydroxyectoine is a superior stress protectant against desiccation for both whole cells and enzymes (Lippert and Galinski, 1992; Louis et al., 1994). This knowledge has subsequently been applied in "anhydrobiotic engineering" of Escherichia coli and Pseudomonas putida (Manzanera et al., 2002, 2004a,b). In addition, a comparative enzyme protection study with heat-stabilizing compounds from extreme thermophiles (Borges et al., 2002) has revealed superb heat-stabilizing properties. However, the biophysical basis of the difference between ectoine and hydroxyectoine, of which only the latter is a good heat and desiccation protectant, has until now not been resolved. The sugars sucrose and trehalose, on the other hand, are well known desiccation protectants in all domains of life and their remarkable function has been linked to the ability to form glasses, which in selected cases ensures conservation of biological functions in an (almost) completely dry state. This phenomenon of "anhydrobiosis" (life without water; Clegg, 2001) is apparent in many higher forms of life (e.g., seeds, resurrection plants, tardigrada, the chironomid Polypedilum vanderplanki). Cytoplasmic glasses are considered one of the main mechanisms of desiccation tolerance (Crowe et al., 1998; Hoekstra et al., 2001; Potts, 2001; Buitink and Leprince, 2004; Ballesteros and Walters, 2011). The outstanding role of disaccharides (possibly in combination with intrinsically disordered proteins, IDPs) has given rise to a number of biophysical models, of which the "water entrapment" (Belton and Gil, 1994; Cottone et al., 2002) and "anchorage" hypothesis (Allison et al., 1999; Francia et al., 2008) are the most comprehensive because they encompass glassformation of solutes and simultaneous entrapment of small water clusters, possibly anchored to critical sites at the interface with biomolecules.

The preservation of biological structures and bioactivity over a long period of time is a main challenge in life science with impacts on medicine, pharmacy and biohybrid technologies (e.g., biosensors). As the halophilic Halomonas elongata is unable to synthesize trehalose and/or sucrose, but appears to convert ectoine into hydroxyectoine in response to heat and water stress, we investigated whether this provides an alternative adaptation strategy for survival of cells and biomolecules in the dry state. This required a biophysical comparison of the glass-forming abilities of ectoine and hydroxyectoine and, in particular, the thermal stability of anhydrous glasses as characterized by the glass transition temperature $(\mathrm{Tg})$. As intermolecular $\mathrm{H}-$ bonding plays an essential role in thermal properties of glasses (Omayu etal., 2008; Zhou et al., 2013), temperature-controlled infrared spectroscopy is widely used to study hydrogen bonds in solids. A compatible approach in the study of anhydrous 


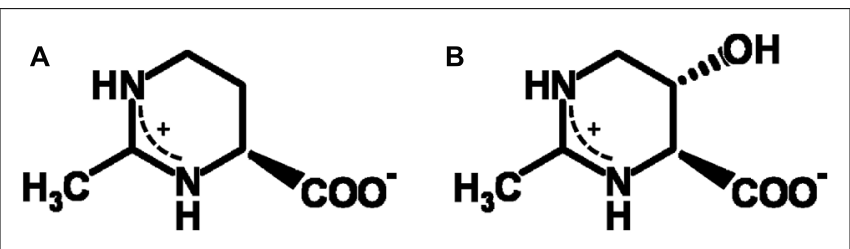

FIGURE 1 | Chemical structure of ectoine (A) and hydroxyectoine (B).

glasses is spin probe electron spin resonance (ESR). As the motion of spin probe molecules is influenced by their intermolecular $\mathrm{H}$-bonding with surrounding molecules, the dynamics of spin probe molecules is obtained from the analysis of the shape of their ESR spectra. Both approaches were used here to characterize differences in glass properties of ectoine and hydroxyectoine.

High molecular immobilization in glasses provides chemical stability of the system due to restricted molecular diffusion. Chemical stability together with structural stability are the main factors which determine survival of organisms in the dry state. The spin probe approach provides an opportunity to obtain information about molecular immobilization and redox activity from the same ESR spectra. Spin probe molecules can participate in different redox-reactions resulting in non-paramagnetic species (Belkin et al., 1987). Therefore, the spin probe approach is a unique technique which allows direct observation of the relationships between structural and chemical stability of dry systems.

\section{MATERIALS AND METHODS CHEMICALS}

Antibiotic broth medium No. 3 (complex medium) was purchased from Oxoid LTD (Hampshire, Great Britain). Pyruvic acid, sucrose, $\mathrm{KH}_{2} \mathrm{PO}_{4}, \mathrm{KOH}$, and $\left(\mathrm{NH}_{4}\right)_{2} \mathrm{SO}_{4}$ were purchased from Roth (Karlsruhe, Germany). D-glucose, $\mathrm{MgSO}_{4} \cdot 7$ $\mathrm{H}_{2} \mathrm{O}$, and $\mathrm{FeSO}_{4} \cdot 7 \mathrm{H}_{2} \mathrm{O}$ were obtained from Merck (Darmstadt, Germany). Trehalose and $\mathrm{NaCl}$ were purchased from Fluka (Buchs, Schwitzerland). Ectoine ( $\geq 99 \%)$ for lactate deydrogenase stress experiments was purchased from bitop AG (Witten, Germany). Hydroxyectoine ( $\geq 99 \%$ ) for lactate dehydrogenase (LDH) stress experiments was isolated from $H$. elongata strain DSM $2581^{\mathrm{T}}$ in our laboratories. Freeze-dried LDH (rabbit muscle) and nicotinamide adenine dinucleotide were purchased from Sigma (Steinheim, Germany). Phosphate buffered saline (PBS) was purchased from AppliChem (Darmstadt, Germany). Ectoine $(\geq 99.0 \%)$ and hydroxyectoine $(\geq 98 \%)$ for spin lable and Fourier transform infrared (FTIR) experiments were purchased from Sigma (USA). Perdeuterated spin probe Tempone- $\mathrm{d}_{16}$ (4-Oxo-2,2,6,6-tetramethylpiperidine- $\mathrm{d}_{16}$-1-oxyl; Figure 7 inset) was a kind gift of Prof. I. Grigoriev (Institute of Organic Chemistry of the Russian Academy of Sciences, Novosibirsk, Russia).

\section{STRAINS, MEDIA, AND CULTIVATION}

Halomonas elongata strain DSM $2581^{\mathrm{T}}$ was obtained from DSMZ (Braunschweig, Germany). Complex media were used for precultures $(5.0 \mathrm{~g} / \mathrm{L}$ peptone, $1.5 \mathrm{~g} / \mathrm{L}$ yeast extract, $1.5 \mathrm{~g} / \mathrm{L}$ "Lab-Lemco" powder, $1.0 \mathrm{~g} / \mathrm{L}$ glucose, $3.68 \mathrm{~g} / \mathrm{L} \mathrm{K} \mathrm{HPO}_{4}, 1.32 \mathrm{~g} / \mathrm{L} \mathrm{K \textrm {K } _ { 2 }} \mathrm{PO}_{4}$ and $150 \mathrm{~g} / \mathrm{L} \mathrm{NaCl} ; \mathrm{pH}$ was adjusted to 7.4). Minimal media MM63 were used for second pre- and for main culture $\left[13.61 \mathrm{~g} / \mathrm{L} \mathrm{KH}_{2} \mathrm{PO}_{4}\right.$, $4.21 \mathrm{~g} / \mathrm{L} \mathrm{KOH}, 0.25 \mathrm{~g} / \mathrm{L} \mathrm{MgSO} 4.7 \mathrm{H}_{2} \mathrm{O}, 1.98 \mathrm{~g} / \mathrm{L}\left(\mathrm{NH}_{4}\right)_{2} \mathrm{SO}_{4}$, $0.0011 \mathrm{~g} / \mathrm{L} \mathrm{FeSO}_{4} \cdot 7 \mathrm{H}_{2} \mathrm{O}, 5 \mathrm{~g} / \mathrm{L}$ glucose, and the required amount of $\mathrm{NaCl}, \mathrm{pH}$ was adjusted to 7.4; Larsen et al., 1987].

Bacterial cultures were grown in shake flasks. Cell growth was tracked by optical density measurement of incubated media at a wavelength of $600 \mathrm{~nm}$. For solute content analysis H. elongata was grown in MM63 with 10 or $15 \% \mathrm{NaCl}(\mathrm{w} / \mathrm{w})$ at 30,40 , and $45^{\circ} \mathrm{C}$, respectively. In addition, to surpass its temperature limit of growth, a thermal shock from 37 to $50^{\circ} \mathrm{C}$ was applied in the mid exponential growth phase. For the determination of desiccation survival, H. elongata was grown in two main cultures MM63 with $15 \% \mathrm{NaCl}$ at $30^{\circ} \mathrm{C}$. In the mid exponential growth phase one of the main cultures was shocked to $50^{\circ} \mathrm{C}$ the other remained at $30^{\circ} \mathrm{C}$. After $4 \mathrm{~h}$ in stationary phase samples were taken for survival experiments.

\section{SOLUTE CONTENT ANALYSIS}

Bacterial cell material was harvested in the stationary growth phase and dried in a rotational vacuum concentrator (SpeedVac) for at least $8 \mathrm{~h}$ at $45^{\circ} \mathrm{C}$ and 10 mbar. Solute extraction was achieved following the Bligh and Dyer protocol (Bligh and Dyer, 1959), as modified by Galinski and Herzog (1990). Homogenized samples $(30 \mathrm{mg}$ ) of dried bacterial biomass were extracted by vigorous shaking $(10 \mathrm{~min})$ with $500 \mu \mathrm{L}$ of modified Bligh and Dyer solution [methanol/chloroform/water 10:5:4 (v/v)]. Following the addition of $130 \mu \mathrm{L}$ chloroform and $130 \mu \mathrm{L}$ water, phase separation was assisted by centrifugation at approximately $9300 \mathrm{~g}$ $(5 \mathrm{~min})$ and the resulting polar upper phase was used for high performance liquid chromatography (HPLC) analysis. For the detection of neutral zwitter-ionic or polar uncharged water soluble compatible solutes an aminopropyl-modified silica column was used (Grom-Sil Amin-1PR, $3 \mu \mathrm{m}, 125 \times 4 \mathrm{~mm}$, LiChrocartSystem, Alltech Grom GmbH). The isocratic eluent was $80 \%$ acetonitrile-water $(\mathrm{v} / \mathrm{v})$ at a flow rate of $1 \mathrm{~mL} / \mathrm{min}$. A combination of UV- and RI-detector was used for peak identification and solute quantification.

\section{DESICCATION-SURVIVAL EXPERIMENT}

Stationary phase samples were diluted with glucose-free medium to an optical density of 0.1 . From this dilution, $100 \mu \mathrm{L}$ were taken to determine the initial cell number (threefold). The same volume $(100 \mu \mathrm{L})$ of each sample was transferred into both a closed $1.5 \mathrm{~mL}$ microcentrifuge tube (undried control) and an open $1.5 \mathrm{~mL}$ microcentrifuge tube. The samples were then subjected to vacuum drying for $3 \mathrm{~h}$ at $45^{\circ} \mathrm{C}$ and $10 \mathrm{mbar}$ in a SpeedVac (RVC 2-25 CD plus, Christ, Osterode am Harz). Subsequently, dried samples (from open microcentrifuge tubes) were rehydrated by adding $1 \mathrm{~mL}$ of glucose-free MM63. Samples were further diluted to $10^{-6}$ or $10^{-7}$ for viable cell number determination. This procedure was repeated three times for every sample. Appropriate dilutions of the samples were plated on a complex medium agar plate with $15 \% \mathrm{NaCl}$. After $48 \mathrm{~h}$ of incubation at $30^{\circ} \mathrm{C}$ colony forming units (CFU) were counted and expressed as percentage of initial cell number. 


\section{CASTING OF SOLUTE GLASS MATRICES}

To cast solute glass matrices $3 \mu \mathrm{L}$ of $2 \mathrm{M}$ solute solution in ultrapure water were placed on a clean polystyrene plate and dried at $60^{\circ} \mathrm{C}$ for $2 \mathrm{~h}$. Transmission light microscopy was used for optical characterization of glass matrices.

\section{LACTATE DEHYDROGENASE (LDH) STRESS EXPERIMENT AND ACTIVITY ASSAY}

Ten microliter of $2 \mathrm{M}$ solute solution or ultrapure water with $0.05 \mathrm{mg} / \mathrm{mL} \mathrm{LDH}$ were placed carefully into separated wells of a 96 well plate. Solidification of solute solutions was accomplished by air drying at $60^{\circ} \mathrm{C}$ for 2,4 , and $6 \mathrm{~h}$. Rehydration to the original volume was accomplished within 2 min of incubation in PBS buffer, pH 7.5 (AppliChem, Darmstadt) at room temperature. The working volume $(200 \mu \mathrm{L})$ for the activity determination of undried (control) and rehydrated LDH solute matrices was obtained by diluting (20-fold) to a final enzyme concentration of $2.5 \mu \mathrm{g} / \mathrm{mL} \mathrm{LDH}$ with $160 \mu \mathrm{L}$ PBS buffer ( $\mathrm{pH} 7.5$ ), $20 \mu \mathrm{L}$ $10 \mathrm{mM}$ pyruvic acid (final concentration $1 \mathrm{mM}$ ) and by addition of $20 \mu \mathrm{L}$ of $7.5 \mathrm{mM} \mathrm{NADH} / \mathrm{H}^{+}$(final concentration of $0.75 \mathrm{mM}$ ) to start the reaction. Decrease in absorbance at $340 \mathrm{~nm}$ was monitored.

\section{SPIN LABEL EXPERIMENTS}

Tempone was added to aqueous solutions of ectoine or hydroxyectoine $(32 \mathrm{mg} / \mathrm{mL})$ in a final concentration of $1 \mathrm{~mol} \%$, so that in a dry state the proportion of (hydroxy) ectoine: Tempone $=100: 1$. At such proportion the concentration broadening of the ESR spectra would not be observed under conditions of uniform distribution of spin probe molecules. Samples of labeled solution $(100 \mu \mathrm{L})$ were spread over chemically inert glass beads (80-110 $\mu$ m diameter) on a glass slide and allowed to dry for 5 days a in a stream of dry air (3\% RH) at room temperature in an airdry box. The dried material was transferred to 2-mm capillaries in an air-dry box $(3 \% \mathrm{RH})$, to prevent rehydration on air, and then flame-sealed.

The capillary with the sample was placed in an ESR quartz tube for spectrum recording. ESR spectra were recorded with an Xband ESR spectrometer (Elexsys model E 500; Bruker Analytik, Rheinstetten, Germany) equipped with a temperature unit using regular air within the temperature range $295-400 \mathrm{~K}$ and liquid $\mathrm{N}_{2}$ for temperatures below $295 \mathrm{~K}$. The spectra were recorded at $5^{\circ}$ increments with equilibration for $1 \mathrm{~min}$ at each temperature. The scan range was $100 \mathrm{G}$ for all spectra. To prevent over-modulation and saturation of ESR signal, the modulation amplitude was $2.5 \mathrm{G}$ for solid-like spectra and $1 \mathrm{G}$ for fluid-like spectra. The microwave power was limited to $5 \mathrm{~mW}$.

\section{FTIR EXPERIMENTS}

Small volumes $(5 \mu \mathrm{L})$ of aqueous solution of ectoine or hydroxyectoine $(32 \mathrm{mg} / \mathrm{ml})$ were dried on circular $\mathrm{CaF}_{2}$ windows $(2 \times 13 \mathrm{~mm})$ in a stream of dry air $(3 \% \mathrm{RH})$ at room temperature in an air-dry box. Although most of the water was removed fast, the samples were further air-dried at 3\% $\mathrm{RH}$ for 5 days in order to achieve equilibrium water potential. Each sample was hermetically sealed between two $\mathrm{CaF}_{2}$ windows using a rubber $\mathrm{O}$-ring and mounted into a temperature-controlled brass cell.
Infrared spectra of dry ectoine and hydroxyectoine were obtained with a Perkin-Elmer series 1725 FTIR spectrometer equipped with an external beam facility to which a Perkin-Elmer IR-microscope was attached. The microscope was equipped with a narrow band mercury-cadmium-telluride liquid nitrogen-cooled IR-detector. The samples between two $\mathrm{CaF}_{2}$ windows were tightly mounted into a temperature-controlled brass cell that was cooled by liquid nitrogen. The temperature of the cell was regulated by a computer-controlled device that activated a liquid nitrogen pump in conjunction with a power supply for heating the cell. The temperature of the sample was recorded separately using two PT-100 elements that were located very close to the sample windows. The optical bench was purged with dry $\mathrm{CO}_{2}$-free air. Spectra were recorded starting with the lowest temperature with a scanning rate of $1.5^{\circ} \mathrm{C} / \mathrm{min}$. The acquisition parameters were: $4 \mathrm{~cm}^{-1}$ resolution, 32 co-added interferograms, 3500-1000 $\mathrm{cm}^{-1}$ wavenumber range.

Spectral analysis and display were carried out using the Infrared Data Manager Analytical software, version 3.5 (Perkin-Elmer). The temperature-induced changes in dry ectoine and hydroxyectoine matrixes were monitored by observing the position of the bands around 1388 and $1088 \mathrm{~cm}^{-1}$. The band around $1388 \mathrm{~cm}^{-1}$ is present in both ectoine and hydroxyectoine, while the band around $1088 \mathrm{~cm}^{-1}$ is present only in hydroxyectoine (Figure 11B). The band positions were calculated as the average of spectral positions ( $n=50)$ at $75 \%$ of the total peak height (Wolkers et al., 1998). Breaks in the temperature dependence of this peak position were determined as a point of intersection of two regression lines below and above the temperature of the break (Figure 12A).

All ESR and FTIR experiments were conducted on the same samples prepared under the same conditions. Each model experiment was repeated at least twice, and the results of the single experiments are presented.

\section{RESULTS \\ INFLUENCE OF WATER AND TEMPERATURE STRESS ON INTRACELLULAR ECTOINE LEVELS}

The moderately halophilic (optimum 3-5\% NaCl) but extremely halotolerant $H$. elongata employs the compatible solutes ectoine/hydroxyectoine for osmotic adaptation by increasing their cytoplasmic concentration in a near-linear fashion. At a salinity of $15 \% \mathrm{NaCl}, H$. elongata experiences severe water stress and, as a consequence, its growth rate is reduced to 0.1 (approximately 25\% of maximum; Dötsch et al., 2008). In contrast to other members of the family Halomonadaceae (e.g., C. salexigens), H. elongata is not able to synthesize the well-known desiccation protectant trehalose, which makes it a good model for investigating the role of hydroxyectoine. As depicted in Figure 2, increase in both temperature and salinity leads to a higher relative proportion of hydroxyectoine. At its maximum growth temperature of $45^{\circ} \mathrm{C}$ and a salinity of $15 \% \mathrm{NaCl}$, the hydroxyectoine level reached $70 \%$ of total ectoines. Although the organism is unable to grow at $50^{\circ} \mathrm{C}$ from inoculum, it was possible to increase the hydroxyectoine level even further by upshock experiments (i.e., raising the temperature in midexponential phase to $50^{\circ} \mathrm{C}$ ). We used the combination of high salinity (15\%) and temperature upshock to simulate a dehydration event and investigated its impact on survival rates of $H$. elongata. 


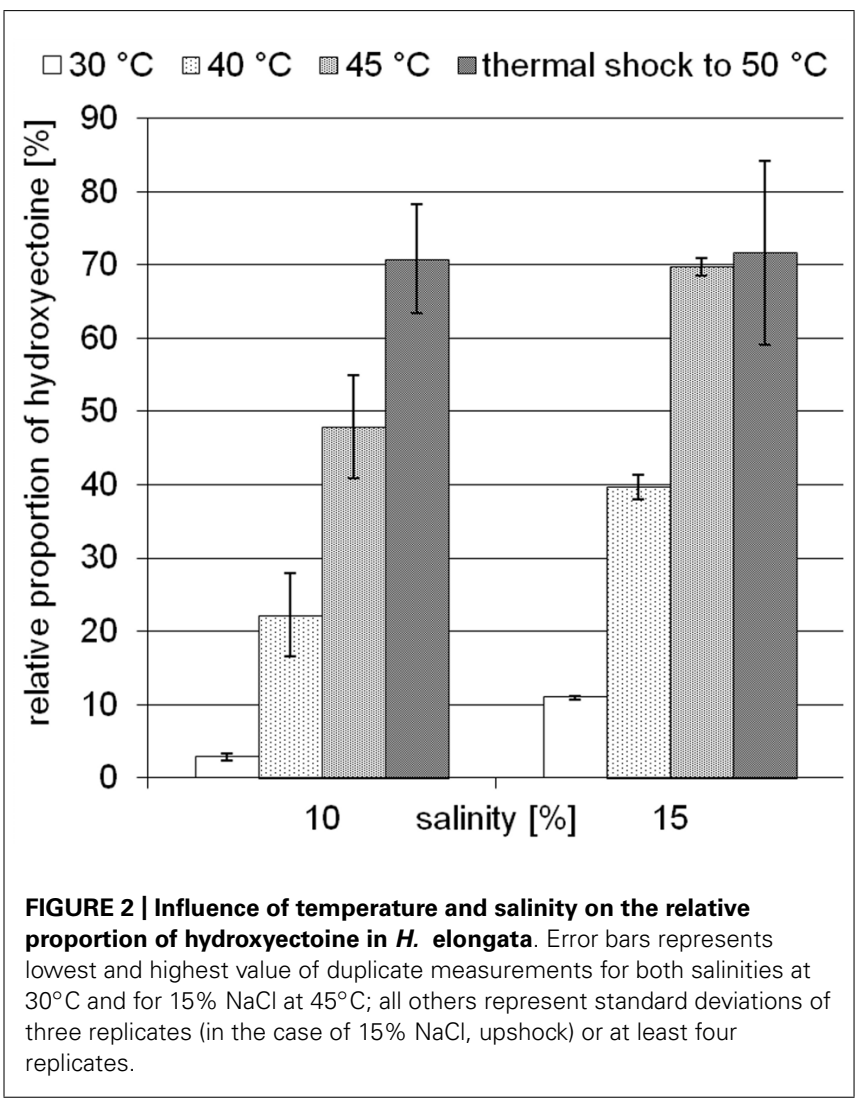

\section{DRY STABILIZATION OF $H$. elongata CELLS AT ELEVATED HYDROXYECTOINE LEVELS}

Two $H$. elongata cultures were grown at $15 \% \mathrm{NaCl}$ and $30^{\circ} \mathrm{C}$ to an optical density of approximately 2, when one of them was heat shocked to $50^{\circ} \mathrm{C}$ (arrow in Figure 3). Subsequently, the solute content of both cultures was analyzed at early stationary phase. It is clearly seen that the applied temperature increase had little effect on the organisms growth and yield. The relative proportion of hydroxyectoine, however, increased from approximately $17 \%$ (at $30^{\circ} \mathrm{C}$ ) to approximately $75 \%$ (at $50^{\circ} \mathrm{C}$; Figure 3, inset), indicating that the conversion of ectoine into hydroxyectoine is enhanced further by temperature upshock. To compare the survival rates of desiccated stationary phase cells, colony-forming units of initial cell numbers (control before drying process), undried controls (from closed vials) and dried samples (vacuum drying for $3 \mathrm{~h}$ at $45^{\circ} \mathrm{C}$ and $10 \mathrm{mbar}$ ) were determined (Figure 4). It was demonstrated that heat-stressed cells with $75 \%$ hydroxyectoine had much higher survival rates. A survival rate of nearly $30 \%$ was achieved as compared to only $4.7 \%$, for untreated cells. Although it cannot be excluded that other adaptational processes may also be responsible for improved survival of heat-shocked cells (see Discussion), we concluded that the hydroxylation of ectoine plays an important part in this improved desiccation survival. Provided that a simple hydroxylation step is indeed able to alter the properties of a common compatible solute (ectoine) in such a way as to provide desiccation protection, then drying of the model enzyme LDH should show a similar response and the

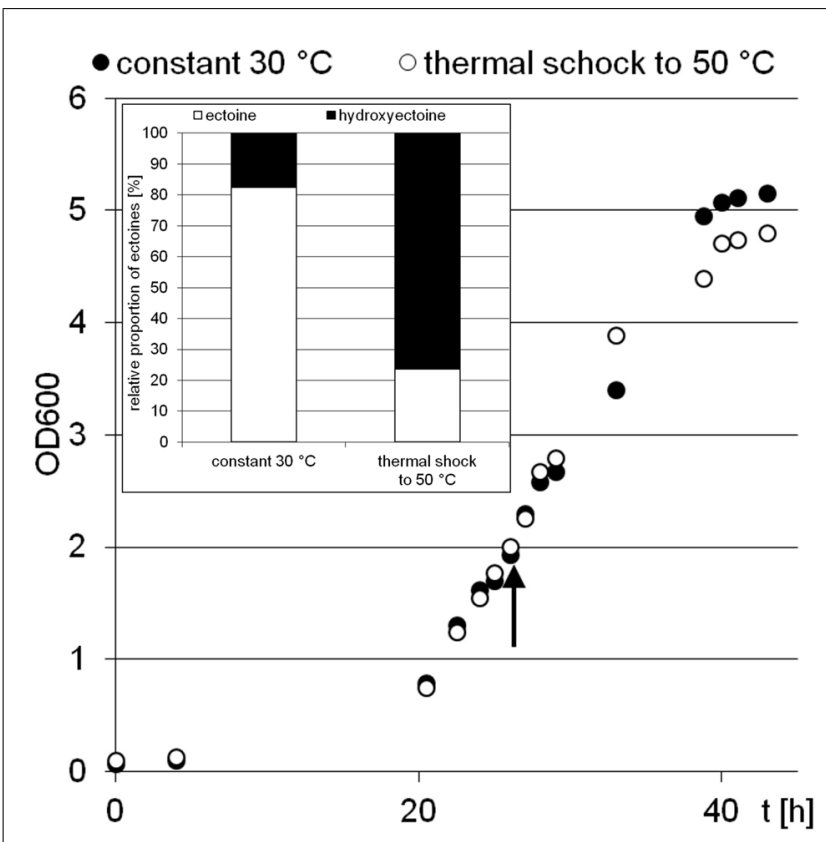

FIGURE 3 | Growth of $\boldsymbol{H}$. elongata in minimal medium MM63 with $15 \%$ sodium chloride. The experiment was performed with two parallel culture, one at constant $30^{\circ} \mathrm{C}$ (black dots), the other with a rapid temperature upshock at $\mathrm{OD} 2$ (arrow) from 30 to $50^{\circ} \mathrm{C}$ (white dots). The inset shows the relative proportions of ectoines in bacterial cells at the early stationary growth phase (point of harvest).

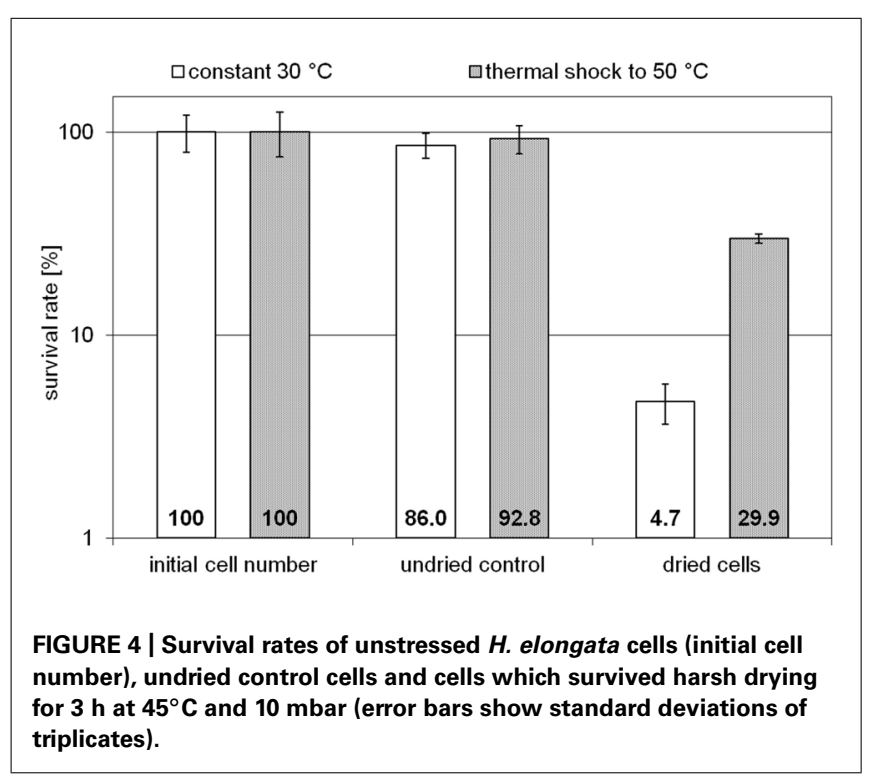

protective effect of hydroxyectoine should compare favorably with the well-known desiccation protectants sucrose and trehalose.

\section{STRESS PROTECTION BY HYDROXYECTOINE DURING HEATING/DRYING OF LACTATE DEHYDROGENASE (LDH)}

Stabilization of model enzyme LDH by compatible solutes against heat and freeze-drying has been investigated before, and for both 


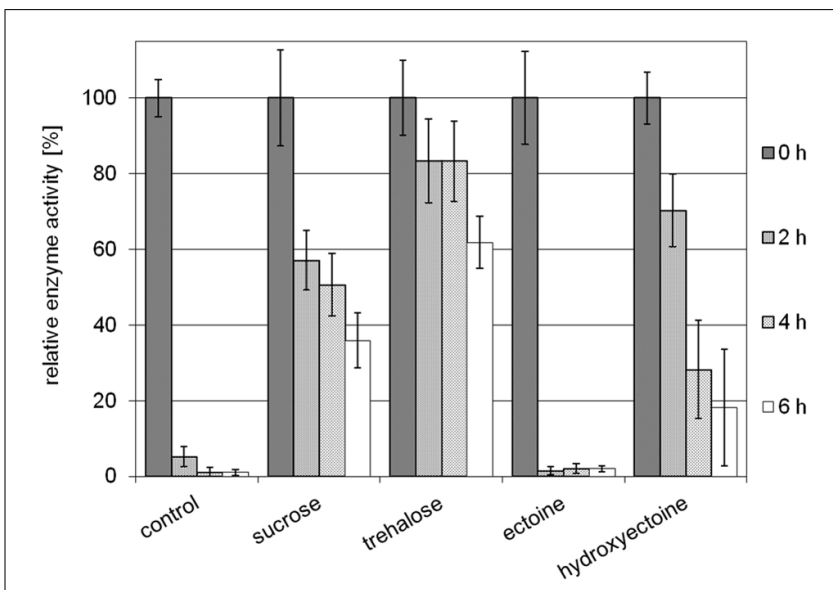

FIGURE 5 | Relative enzyme activities of LDH after prolonged air drying at $60^{\circ} \mathbf{C}$ for $\mathbf{2 , 4}$, and $\mathbf{6 ~ h}$. Activities of unstabilized enzyme (control) and of enzyme protected by sucrose, trehalose, ectoine, and hydroxyectoine, respectively, are shown (error bars show standard deviations of at least four to a maximum of six replicates).

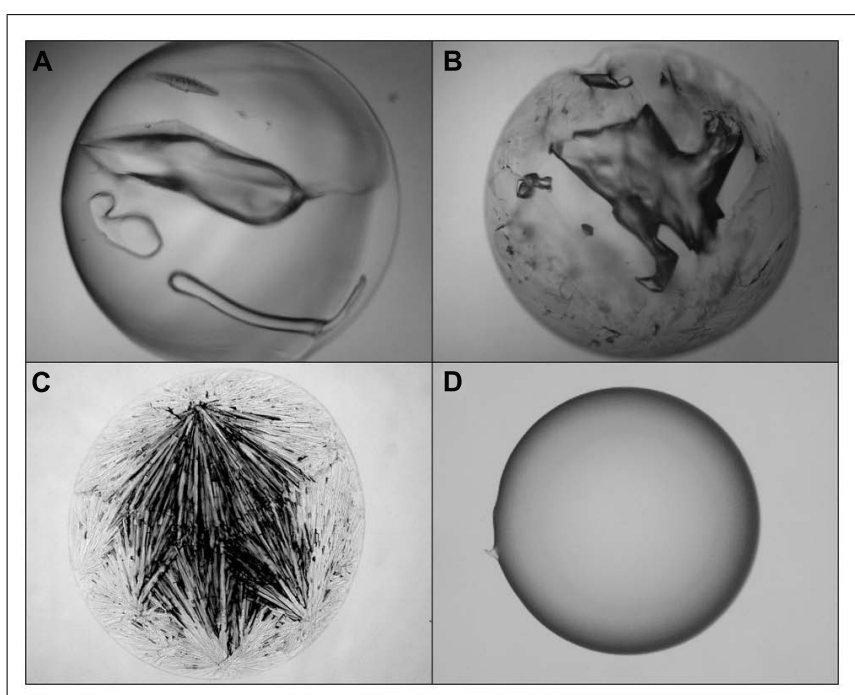

FIGURE 6 | Light microscopic photographs of solute matrices which were casted from $2 \mathrm{M}$ solutions of trehalose (A), sucrose (B), ectoine (C), and hydroxyectoine (D) by air drying of $10 \mu \mathrm{L}$ at $60^{\circ} \mathrm{C}$ for $2 \mathrm{~h}$.

stress factors the superiority of hydroxyectoine over ectoine has been demonstrated (Lippert and Galinski, 1992; Borges et al., 2002). Here a small volume $(10 \mu \mathrm{L})$ of $\mathrm{LDH}$ at a concentration of $0.05 \mathrm{mg} / \mathrm{mL}$ in $2 \mathrm{M}$ solutes (ectoine, hydroxyectoine, sucrose, and trehalose, respectively) was exposed to air-drying at $60^{\circ} \mathrm{C}$. The dried protein was subsequently diluted in buffer and checked for residual activity. As shown in Figure 5, the unprotected enzyme loses approximately $95 \%$ of its original activity after $2 \mathrm{~h}$ of drying. Prolonged drying destroyed activity almost completely. It is worthy of note that the presence of ectoine had no stabilizing effect under the conditions employed, whereas hydroxyectoine after $2 \mathrm{~h}$ drying at $60^{\circ} \mathrm{C}$ displayed a residual activity of approximately $70 \%$, which lies between the values of sucrose $(58 \%)$ and trehalose $(83 \%)$ as benchmarks. Upon further drying, however, the stabilizing effect of hydroxyectoine declined more rapidly than with disaccharides. Nonetheless the observed differences between ectoine (no stabilization) and hydroxyectoine (on a par with disaccharides after $2 \mathrm{~h}$ of drying) are remarkable and put hydroxyectoine into the same category as the glass-forming disaccharides. We therefore expanded our comparison of both ectoines to include their glass-forming abilities.

\section{GLASS-FORMING ABILITIES OF ECTOINES}

A simple glass-casting experiment from $2 \mathrm{M}$ solution at $60^{\circ} \mathrm{C}$ disclosed a striking difference between both ectoines (Figures 6C,D). While hydroxyectoine formed a clear and transparent solid, visual examination of ectoine samples revealed an inhomogeneous structure with crystalline inclusions, indicating a mixture of glassy and crystalline states. It can also be seen that the benchmark glass-formers sucrose and trehalose formed solids with cracks under the drying conditions employed (Figures 6A,B). These were never observed with solid hydroxyectoine samples. Thus it can be concluded that hydroxyectoine, in contrast to ectoine, is a good glass

former and that this property is probably related to the superior desiccation protection of hydroxyectoine on biological structures.

\section{SPIN PROBE STUDY OF GLASS PROPERTIES OF DRY ECTOINE AND HYDROXYECTOINE}

Hydrogen bonds and packing density are the key factors which determine the properties of anhydrous glasses (Omayu et al., 2008; Wang et al., 2009; Zhou et al., 2013). Spin probe Tempone as a reporter molecule was used to study the glass properties of dry ectoine and hydroxyectoine. Tempone is a small watersoluble stable free radical, which has the shape of a sphere with a radius around $3 \AA$. It has one ketone group $>\mathrm{C}=\mathrm{O}$ (Figure 7, inset), which can be an acceptor for $\mathrm{H}$-bonds. The uncoupled electron nitroxide group $\mathrm{N}-\mathrm{O}$ is surrounded by 4 bulky methyl groups (Figure 7, inset) and is probably less available for $\mathrm{H}$ bonding. One molecule of ectoine can provide two donors for hydrogen bonds - two N-H groups from ring $\mathrm{N}$ - and one acceptor $\mathrm{C}=\mathrm{O}$ from $\mathrm{COO}^{-}$group (Figure 1). Hydroxyectoine has one additional potential donor. This is a hydroxyl group $-\mathrm{OH}$, which is attached to the heterocyclic ring (Figure 1). The $\mathrm{H}$ bonds between $>\mathrm{C}=\mathrm{O}$ of Tempone and $\mathrm{N}-\mathrm{H}$ groups of both ectoine and hydroxyectoine are less strong than the $\mathrm{H}$-bond between $>\mathrm{C}=\mathrm{O}$ of Tempone and $\mathrm{OH}$ group of hydroxyectoine because oxygen is more electronegative than nitrogen. Being hydrogen-bonded with ectoine and hydroxyectoine, the spin probe molecule moves collectively with the solute molecules as long as such H-bonds exist. Tempone has an ESR spectrum consisting of three lines due to hyperfine interactions of its uncoupled electron with nitrogen spin (Knowles et al., 1976). Because of spectral anisotropy, ESR spectra of spin probes are sensitive to motion and are, therefore, suitable for a temperature-dependent study of molecular immobilization in a dry matrix caused by H-bonds. 


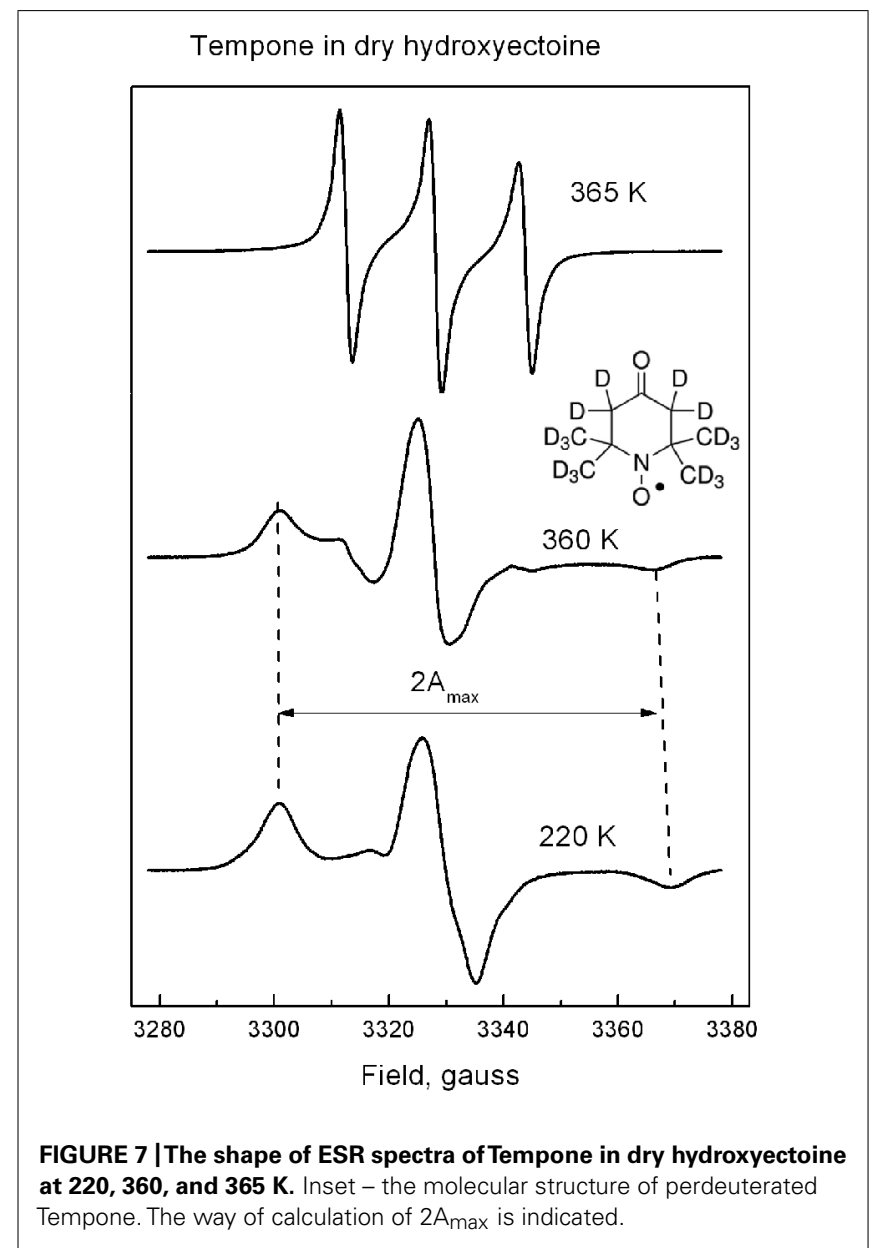

Tempone in dry hydroxyectoine has a solid-like spectrum up to $360 \mathrm{~K}$ (Figure 7). This spectral shape is typical for highly immobilized spin probe molecules. The distance between outer extremes $2 \mathrm{~A}_{\max }$ (Figure 7) is used to characterize the degree of immobilization of the nitroxide moiety of the spin probe (Knowles et al., 1976). Above $360 \mathrm{~K}$ a sudden change in the spectral shape is observed (Figure 7). The spectrum of Tempone at $365 \mathrm{~K}$ in Figure 7 has three equidistant narrow lines. Such a spectral shape is typical for fast isotropic rotation of the spin probe molecule (Knowles et al., 1976).

Figure 8 shows that $2 \mathrm{~A}_{\max }$ of Tempone spectra decreases slowly up to $290 \mathrm{~K}$, after which the rate of disordering increases, but the spin probe remains immobile up to $360 \mathrm{~K}$. Obviously, the break in the temperature dependence of $2 \mathrm{~A}_{\max }$ is caused by some structural rearrangements in the solvent matrix (Dzuba et al., 1993, 2005). Above $360 \mathrm{~K}, 2 \mathrm{~A}_{\max }$ cannot be determined because the spectrum becomes isotropic (Figure 7). The ESR spectrum is the first derivative of the absorption spectrum. To estimate the number of paramagnetic centers in a sample, the ESR spectrum has to be double-integrated to obtain the area under the absorption peak (Figure 8, inset). The double integral of the ESR spectra is called integrated intensity. The onset of a sharp decrease in integrated intensity coincides with the point of fast isotropic rotation at $360 \mathrm{~K}$. Such an increase of the molecular freedom may result

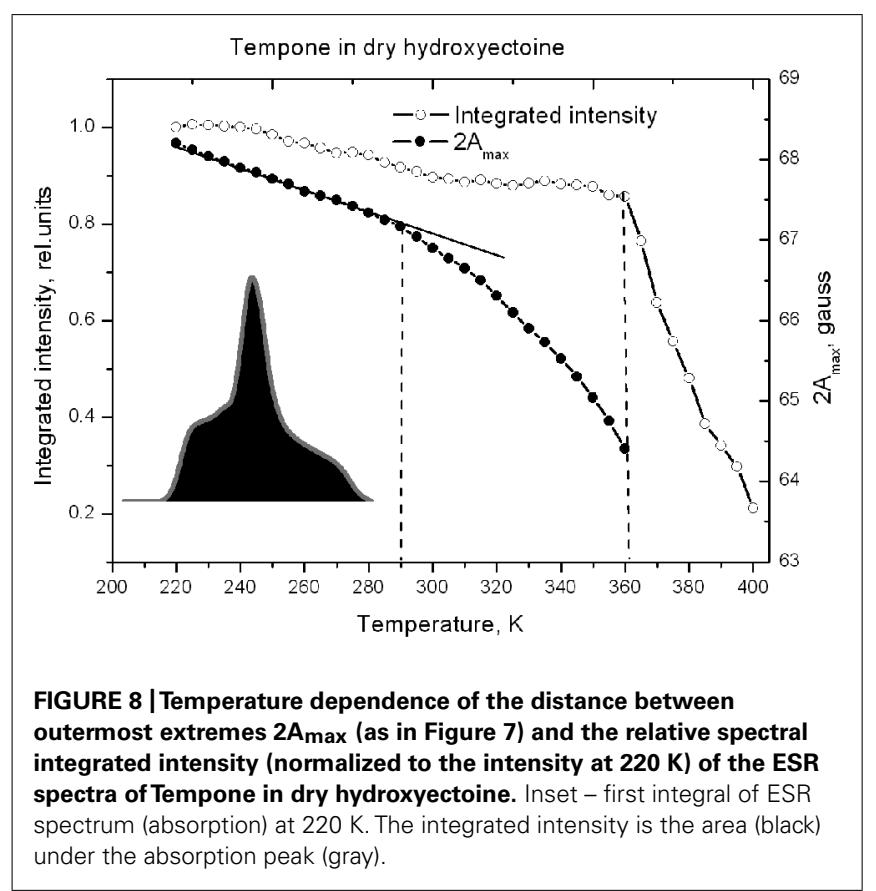

from the breaking of hydrogen bonds between the ketone group of Tempone and the hydroxyl groups of hydroxyectoine.

Figure 9A shows the shape of Tempone spectra in dry ectoine and hydroxyectoine at $220 \mathrm{~K}$. The shapes of the spectra are different. The spectrum from hydroxyectoine was subtracted from that in dry ectoine after spectral titration (adjustment of spectral position and amplitudes). The difference spectrum is a singlet (Figure 9B). The spectrum subtraction shows that the ESR spectrum of Tempone in ectoine is the superposition of a solidlike triplet (as in hydroxyectoine) and a singlet. The solid-like triplet spectrum (as in Figures 7 and 9A) is caused by Tempone molecules, which are spatially separated (i.e., in a glassy state of ectoine). Singlet spectra (Figure 9B) are caused by spin exchange between highly concentrated Tempone molecules (Knowles et al., 1976). This results from Tempone molecules, which are excluded from crystalline ectoine and therefore locally concentrated. It is therefore possible to conclude that under the conditions employed (5 days of air-drying at room temperature), solid ectoine samples are a mixture of glassy and crystalline states.

The superposition of singlet and triplet in Tempone spectra in dry ectoine does not allow the correct determination of $2 \mathrm{~A}_{\max }$. However, it is still possible to characterize the temperatureinduced dynamic changes in Tempone spectra in dry ectoine at a semi-quantitative level by plotting the ratio of the heights of the positive peaks of the low-field narrow line $\mathrm{H}_{+1}$ and the central line $\mathrm{H}_{0}$ (arrows in Figure 9C) against temperature $\left(\mathrm{H}_{+1} / \mathrm{H}_{0}\right.$ vs. temperature). The central line $\mathrm{H}_{0}$ is a superposition of central lines from both solid-like and fluid-like spectra, while the low-field narrow line $\mathrm{H}_{+1}$ represents only the fluid-like spectrum (Figure 9C). $\mathrm{H}_{+1} / \mathrm{H}_{0}$ is an approximate estimation of the proportion of the fluid-like spectral component. Subtraction of the Tempone spectrum of ectoine at $300 \mathrm{~K}$ from that at $340 \mathrm{~K}$ (Figure 9D) shows the 

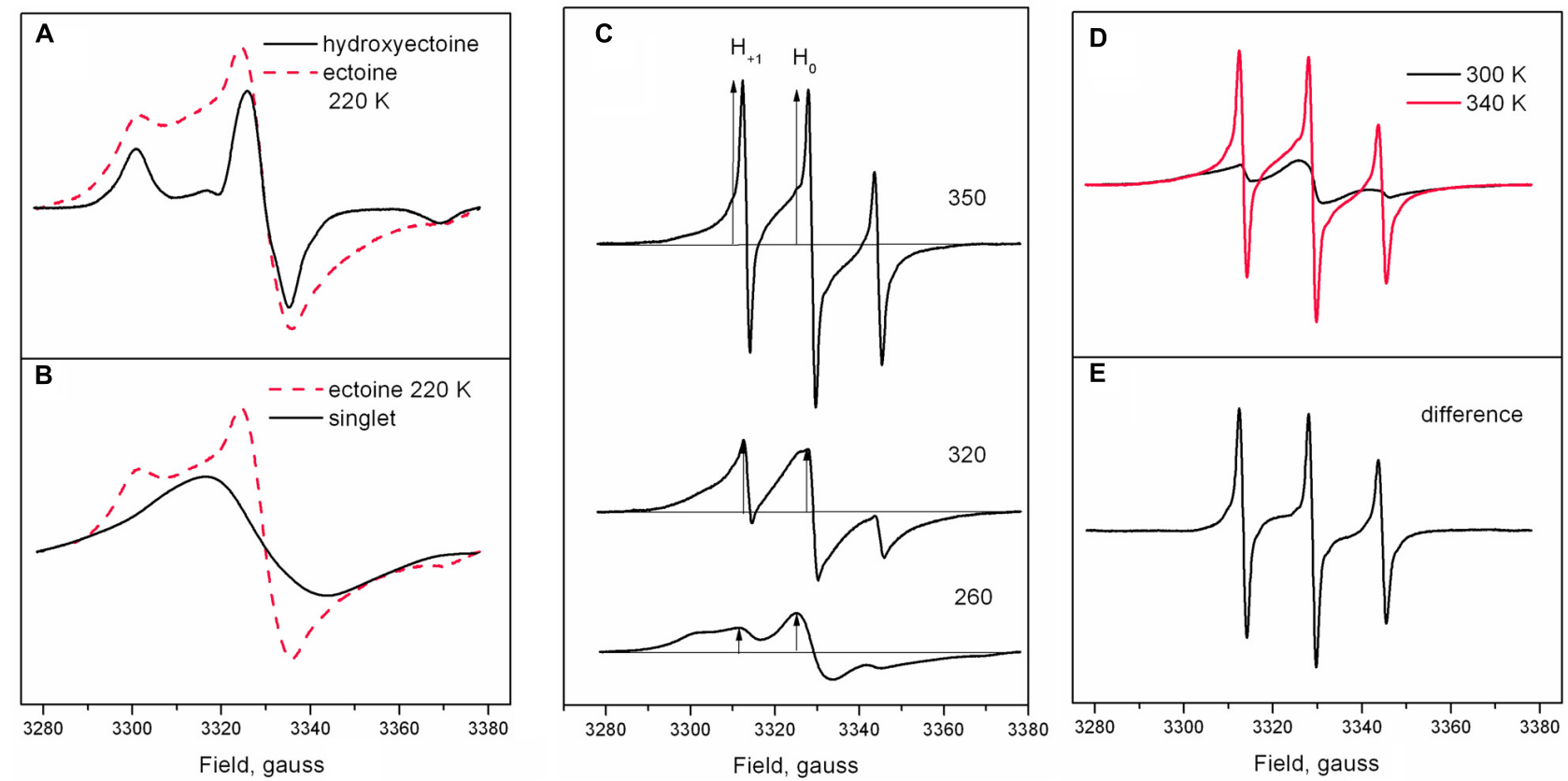

FIGURE 9 | (A) Tempone spectra from dry ectoine and hydroxyectoine at $220 \mathrm{~K}$ adjusted for spectral intensity and peak position; (B) a singlet obtained by subtraction of Tempone spectrum in

hydroxyectoine from Tempone spectrum in ectoine as in (A); dashed red line is a Tempone spectrum in ectoine. (C) Tempone spectra in dry ectoine at different temperatures. Arrows indicate the position of the central line $\mathrm{H}_{0}$ and narrow low-field line $\mathrm{H}_{+1}$. (D) Tempone spectra in dry ectoine at 300 and $340 \mathrm{~K}$ (red line), adjusted for spectral intensity and line position; (E) the difference between spectra in (D). narrow-line spectrum, which has a shape typical for fast isotropic motion of the spin probe molecule (Figure 9E). Figures 9C-E clearly demonstrate the gradual increase of the fluid-like component of the spectra and thus also the increase in the proportion of the freely rotating Tempone molecules in the sample.

Figure 10 shows the temperature-induced changes in $\mathrm{H}_{+1} / \mathrm{H}_{0}$ of Tempone spectra in dry ectoine. In contrast to hydroxyectoine (Figure 8), the fluid-like component of Tempone spectra from ectoine appears already around $240-250 \mathrm{~K}$ as the increase of $\mathrm{H}_{+1} / \mathrm{H}_{0}$, but the main changes occur between 270 and $320 \mathrm{~K}$. At $\mathrm{T}>320 \mathrm{~K}$ the spectral shape is presented mainly by a narrow line isotropic spectrum as in Figure 9C top. This point of change $(320 \mathrm{~K})$ again coincides with the onset of a decrease in integrated intensity of ESR spectra.

Comparison of data in Figures $\mathbf{8}$ and $\mathbf{1 0}$ suggests that $\mathrm{H}$-bonds between Tempone ( $>\mathrm{C}=\mathrm{O}$ ) and hydroxyectoine exist up to $360 \mathrm{~K}$ $\left(87^{\circ} \mathrm{C}\right)$ and are completely broken above this temperature allowing free rotation of spin probe molecules. In ectoine the break in hydrogen bonds between Tempone and solute molecules begins below room temperature and is completed at $320 \mathrm{~K}\left(47^{\circ} \mathrm{C}\right)$. These data show that hydroxyectoine glasses are more thermostable than ectoine glasses.

\section{FTIR STUDY OF SOLID MATRIX OF ECTOINE AND HYDROXYECTOINE}

Spin probe ESR shows only interactions between guest molecule and solute molecules. Temperature-controlled infrared spectroscopy provides information about $\mathrm{H}$-bonding intra- and intermolecular interactions between solute molecules. The FTIR study

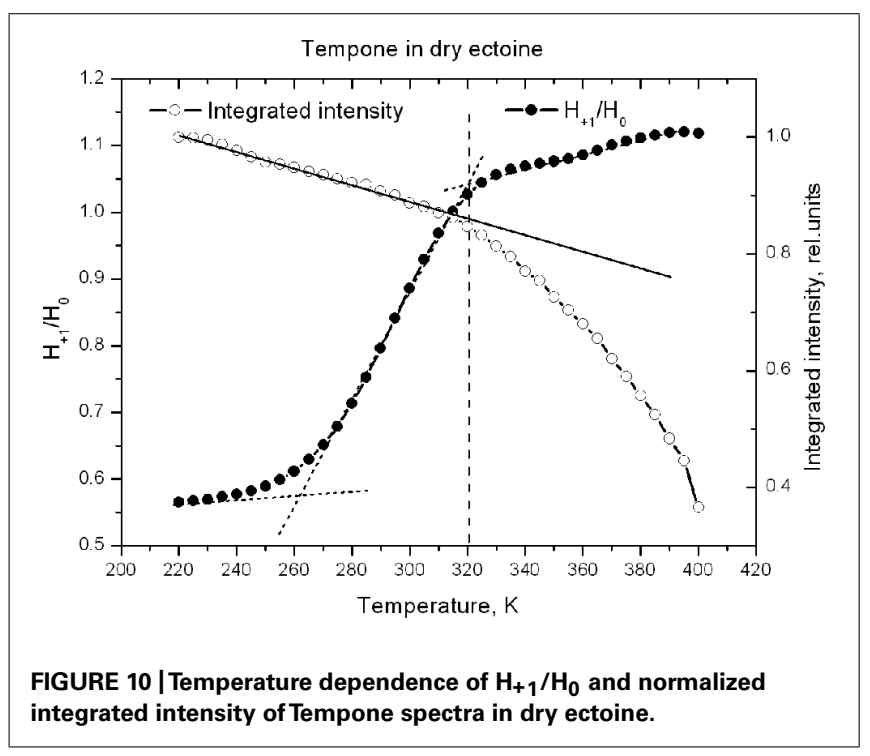

of dry ectoine and hydroxyectoine was performed parallel to the spin probe study. Visually, partial macro-crystallization of dry ectoine was observed. For IR spectra recording an amorphous area, $500 \mu \mathrm{m} \times 500 \mu \mathrm{m}$ in size, was selected under attached PerkinElmer IR- microscope. This partial crystallization of ectoine had already been concluded from the shape of ESR spectra of Tempone in dry ectoine (Figure 9). In the case of hydroxyectoine, no visual crystallization of the dry solid was observed under the microscope. 
Figure 11A shows the IR spectra of dry amorphous ectoine and hydroxyectoine. Both spectra contain broad overlapping bands in the hydrogen stretching region $\mathrm{N}-\mathrm{H}, \mathrm{C}-\mathrm{H}$, and $\mathrm{N}-\mathrm{H}\left(>2500 \mathrm{~cm}^{-1}\right)$ and a set of narrow lines in the finger print region (Figure 11B in details). The $\mathrm{OH}$ stretching vibration band around $3300 \mathrm{~cm}^{-1}$ as a function of temperature was previously used to determine $\mathrm{Tg}$ in dry sugars and their mixtures (Wolkers et al., 1998, 2004; Kets et al., 2004; Imamura et al., 2006). The overlapping of different broad peaks in the hydrogen stretching region $2500-3500 \mathrm{~cm}^{-1}$ in ectoine and hydroxyectoine spectra (Figure 11A) does not allow the use of $\mathrm{OH}$ stretching band at around $3300 \mathrm{~cm}^{-1}$ for characterization of glasses formed by these compounds. The fingerprint region of the FTIR spectra contains several peaks, which are shown in detail in Figure 11B. The identification of peaks in the fingerprint region is difficult and needs additional information (Coates, 2000). The fingerprint area of FTIR spectra from dry hydroxyectoine has some additional peaks which are not present in ectoine, and some similar peaks (Figure 11B). The differences are caused by the presence of additional $\mathrm{OH}$ group in hydroxyectoine, which is attached to the heterocyclic ring.

The information obtained from ESR experiments (Figures 8 and 10) can be used for assignment of some bands in the fingerprint region of the spectra in Figure 11B. Our spin probe study showed that the $\mathrm{H}$-bonds between $>\mathrm{C}=\mathrm{O}$ of Tempone and $\mathrm{OH}$ group of dry hydroxyectoine are broken at approximately $360 \mathrm{~K}$. The inspection of the wavenumber-temperature dependences of all major peaks in the fingerprint area of hydroxyectoine showed that only the bands around 1088 and $1388 \mathrm{~cm}^{-1}$ (Figure 11B) have a break at approximately $360 \mathrm{~K}$ (Figures 12A,B). The peak around $1088 \mathrm{~cm}^{-1}$ does not exist in the ectoine spectrum, and can thus be attributed to $\mathrm{OH}$ group attached to heterocyclic ring in hydroxyectoine. On the other hand, the FTIR spectrum of dry ectoine has the same band around $1388 \mathrm{~cm}^{-1}$ as hydroxyectoine (Figure 11B) and can be assigned to $\mathrm{NH}$ groups in both ectoine and hydroxyectoine. However, the break in the wavenumber-temperature dependence

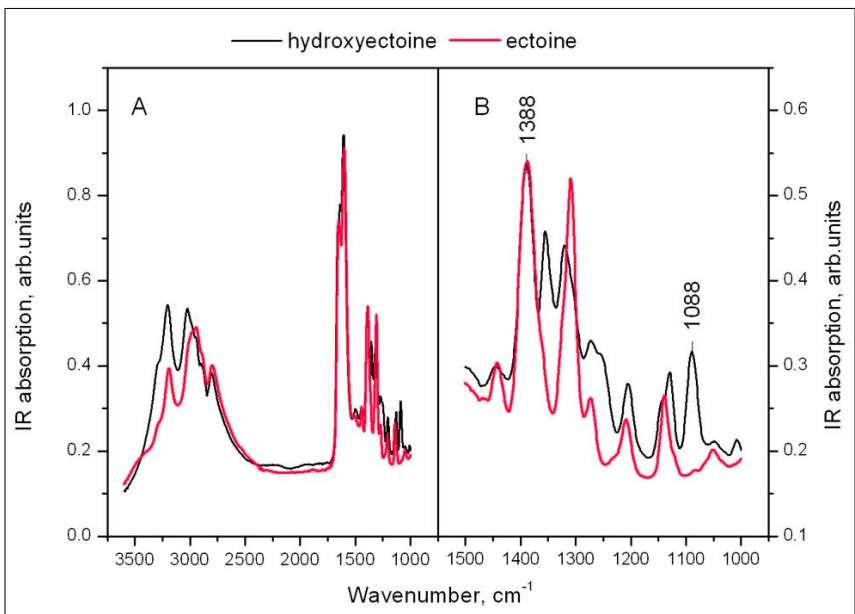

FIGURE 11 | (A) FTIR spectra of dry amorphous ectoine (red line) and hydroxyectoine at room temperature. The intensity of the spectra are normalized to the height of the peak at $1600 \mathrm{~cm}^{-1}$; (B) enlarged finger print region of FTIR spectrum.

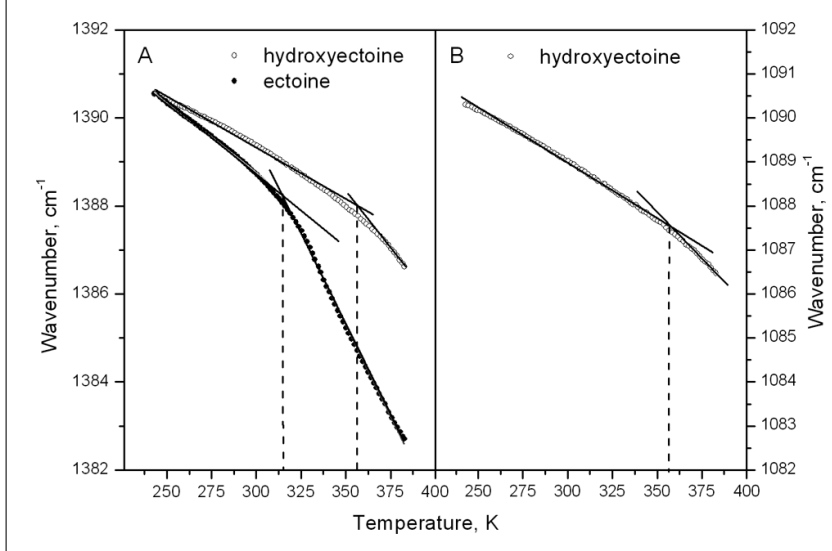

FIGURE 12 | Temperature dependence of the frequency vibration at $1382-1392 \mathrm{~cm}^{-1}$ in dry ectoine and hydroxyectoine $(A)$ and at $1082-1092 \mathrm{~cm}^{-1}$ in dry hydroxyectoine (B).

for this band in ectoine occurs not at $360 \mathrm{~K}$, as in hydroxyectoine, but at approximately $320 \mathrm{~K}$ (Figure 12A). This coincides with the temperature at which all Tempone molecules start free rotation (Figures 9 and 10). The fact that IR inflections of hydroxyectoine are less distinct, is explained by experimental limitations (i.e., upper temperature limit of $100^{\circ} \mathrm{C}$ und, therefore, fewer data points beyond $\mathrm{Tg}$ ) and higher strength of $\mathrm{OH}$ hydrogen bonds resulting in a less pronounced slope of the wavenumber vs. temperature plot.

\section{DISCUSSION \\ PROTEIN STABILIZATION EFFECTS OF COMPATIBLE SOLUTES IN SOLUTION}

Compatible solutes (organic osmolytes) of halophilic bacteria have long been known as versatile stress-protecting compounds, in particular for the stabilization of proteins and whole cells. The molecular nature of their stabilizing function has been explained by preferential interaction with water and subsequent exclusion from a proteins hydration shell (Arakawa and Timasheff, 1985). This original concept has been expanded by others, who revealed that the unfavorable interaction of the peptide backbone is the main driving force for this stabilization phenomenon, named the "osmophobic effect" (Bolen and Baskakov, 2001). In order to elucidate the molecular features which make a solute a stabilizing compound, a quantitative solvation model was proposed (Street et al., 2006), in which backbone/solute interaction energy is a function of the interactants' polarity and surface area. On the basis of this model, we are now able to quantify the chemical features, which make a solute "compatible" and rank them according to their protein stabilization in solution. The superiority of hydroxyectoine over ectoine in desiccation protection of cells and biomolecules has, however, so far not been explained.

\section{ESR STUDIES PROVE SUPERIOR GLASS PROPERTIES OF HYDROXYECTOINE}

The presented ESR data show that dry hydroxyectoine has considerable advantages as a matrix for guest molecules over ectoine. Hydroxyectoine forms stable glasses, while ectoine is more prone 
to crystallization. The difference is caused by one additional $\mathrm{OH}$ group in hydroxyectoine. This group reduces the degree of molecule symmetry and thus probably prevents the molecules from tight packing (Wang et al., 2009). This creates the space for optimized orientation of molecules for $\mathrm{H}$-bonding, the formation of which needs not only the presence of donor and acceptor, but also specific geometry (Steiner, 2002). The more bulky shape of hydroxyectoine in dry matrix provides better conditions for $\mathrm{H}$ bonding with other molecules than the more symmetric ectoine, which has a tendency to aggregate and crystallize. As a result, the additional hydroxyl provides more possibilities for $\mathrm{H}$-bonds by increasing the number of donors, the strength of intermolecular interactions and the energy barrier for the reorganization of the molecules. It has already been shown that the presence of hydroxyl groups increases thermal stability of glasses (Omayu et al., 2008; Zhou et al., 2013).

The large temperature difference between molecular fluidizing in ectoine $(320 \mathrm{~K})$ and hydroxyectoine $(360 \mathrm{~K})$ of 40 degrees, as shown by ESR and FTIR studies (Figures 8, 10, and 12), results from different types of intermolecular $\mathrm{H}$-bonds that cause molecular immobilization. In ESR experiments, the motional behavior of Tempone in hydroxyectoine is determined by the strongest $\mathrm{H}$ bonds between $\mathrm{CO}$ of Tempone and $\mathrm{OH}$ of the solute. In ectoine, motional behavior of Tempone is determined by weaker $\mathrm{H}$-bonds with $\mathrm{NH}$ groups of the solute.

Stronger H-bonds provide not only better structural stability but also chemical stability of Tempone molecules in dry hydroxyectoine. Chemical stability of dry material is associated with molecular immobilization, which considerably reduces the probability of diffusion-controlled reactions. The probability of chemical reactions increases above glass transition (Craig et al., 2001). The spin probe approach provides an opportunity to obtain information about molecular immobilization and redox activity from the same ESR spectra. Spin probe molecules can participate in different redox-reactions resulting in non-paramagnetic species. The most probable are reduction to hydroxylamine and irreversible reaction with other radicals to stable non-radical products (Belkin et al., 1987). In the case of redox conversion of spin probe molecules, the number of paramagnetic species decreases resulting in the decrease of the integral intensity of the spectra (Figures 8 and 10).

The integrated intensity of the ESR spectra of Tempone in dry hydroxyectoine does not significantly change up to the point of breaking H-bonds between 360 and $365 \mathrm{~K}$. Above this temperature, the integrated intensity of the spectra sharply decreases (Figure 8). This coincides with the transformation of the spectral shape of Tempone to fluid-like type (Figure 7). The break in the temperature dependence of the integrated intensity of Tempone spectra in dry ectoine was observed at $320 \mathrm{~K}$ (Figure 10). As in the case of hydroxyectoine, the redox conversion of Tempone in dry ectoine sharply increases when spin probe molecules obtain a motional freedom similar to that in a fluid phase (Figure 9E). Such motional freedom was obviously caused by the break of $\mathrm{H}$-bonds $>\mathrm{C}=\mathrm{O} . . . \mathrm{HN}$ (>CO belongs to Tempone, $\mathrm{NH}$ belongs to ectoine). Clearly, redox conversion of spin probe molecules becomes possible when they obtain motional freedom. The fluid-type spectrum of Tempone indicates the possibility of translational diffusion and increased probability of chemical reactions. Therefore, the presence of hydroxyectoine in the dry cytoplasm of anhydrobiotic organisms would improve the structural and chemical stability of glasses as a result of increased number and strength of $\mathrm{H}$-bonds between molecules.

\section{FTIR STUDIES CONFIRM GLASS TRANSITION TEMPERATURES OF ECTOINE AND HYDROXYECTOINE}

The broad region of $\mathrm{OH}$ stretching vibrations around $3380 \mathrm{~cm}^{-1}$ in FTIR spectra is commonly used to determine Tg in dry sugars and their mixtures (Wolkers et al., 1998, 2004; Kets et al., 2004; Imamura et al., 2006). Ectoine does not contain OH groups and therefore does not contain a distinct band in this region (Figure 11A). Hydroxyectoine contains one $\mathrm{OH}$ group attached to the heterocyclic ring, and the FTIR spectrum is expected to display the band around $3380 \mathrm{~cm}^{-1}$ from $\mathrm{OH}$ stretching vibrations as for dry sugars. However, only a weak shoulder is present in this region in the FTIR spectrum of hydroxyectoine (Figure 11A). Obviously, the broad bands from $\mathrm{NH}$ stretching and $\mathrm{CH}$ stretching vibrations $\left(2800-3200 \mathrm{~cm}^{-1}\right)$ mask the $\mathrm{OH}$ band at $3380 \mathrm{~cm}^{-1}$. Close inspection of bands within the fingerprint region helped to find other bands, which can be used to characterize glass transition in dry (hydroxy)ectoine. The decrease of the wavenumber with temperature for both bands at 1388 and $1088 \mathrm{~cm}^{-1}$ (Figures 12A,B) is characteristic for bending deformations of the functional groups, which are involved in H-bonding (Vanderkooi et al., 2005). All data together allow the assignment of the band around $1388 \mathrm{~cm}^{-1}$ to bending deformations of $\mathrm{NH}$ groups of the ring, and the band around $1088 \mathrm{~cm}^{-1}$ to bending deformations of $\mathrm{OH}$ attached to the heterocyclic ring. The same position of the break of the temperature dependence for $\mathrm{OH}$ and $\mathrm{NH}$ bending vibrations in hydroxyectoine $(360 \mathrm{~K}$, Figures 12A,B) indicates that in this dry matrix $\mathrm{NH}$ groups form intermolecular $\mathrm{H}$-bonds with $\mathrm{OH}$ groups. In ectoine, $\mathrm{OH}$ groups are absent and such $\mathrm{H}$-bonds are not possible. Weaker intermolecular $\mathrm{H}$-bonds between $\mathrm{NH}$ and $\mathrm{CO}$ of $\mathrm{COO}^{-}$group and, even weaker still, $\mathrm{H}$-bonds between $\mathrm{NH}$ groups determine the lower position of the break in the temperature dependence of the wavenumber of $\mathrm{NH}$ bending vibration in dry ectoine (Figure 12A).

In conclusion, it can be said that FTIR data corroborated the ESR observations, which demonstrate a remarkable difference in $\mathrm{Tg}$ of approximately $40^{\circ} \mathrm{C}$ between ectoine and hydroxyectoine. The high $\mathrm{Tg}$ of the latter $\left(87^{\circ} \mathrm{C}\right)$ places hydroxyectoine somewhere between sucrose $\left(65^{\circ} \mathrm{C}\right)$ and trehalose $\left(117^{\circ} \mathrm{C}\right)$.

\section{RESPONSE OF H. elongata TO SALT AND TEMPERATURE STRESS}

Earlier studies have shown increasing hydroxyectoine levels in response to salinity and temperature (Wohlfarth et al., 1990; Severin et al., 1992) in the Halomonadaceae. This upregulation of hydroxyectoine synthesis has subsequently been investigated in greater depth with the related $C$. salexigens. Under comparable growth conditions $(1.5 \mathrm{M} \mathrm{NaCl}=8.7 \%$ and $2.5 \mathrm{M} \mathrm{NaCl}=14.5 \%$, at a temperature of $45^{\circ} \mathrm{C}$ ), relative proportions of hydroxyectoine of approximately 60 and $70 \%$ respectively have been reported (Garcia-Estepa etal., 2006; Rodríguez-Moya etal., 2013). By genetic manipulation (transcriptional fusion, gene duplication, 
and overexpression on vector), this proportion was increased even further to $77 \%\left(8.7 \% \mathrm{NaCl}, 37^{\circ} \mathrm{C}\right)$, albeit at the expense of a reduced growth rate (Rodríguez-Moya et al., 2013). As demonstrated by Figure 2, $H$. elongata grown at $15 \% \mathrm{NaCl}$ similarly increases its relative proportion of hydroxyectoine in response to both salinity and temperature, with a maximum of approximately $70 \%$ at the highest tolerated growth temperature of $45^{\circ} \mathrm{C}$. However, we report here for the first time that a sudden temperature upshift to $50^{\circ} \mathrm{C}$ (beyond the organism's temperature maximum) also leads to a rapid increase in the proportion of hydroxyectoine (up to 75\%). Surprisingly, such a temperature shift, when applied in the exponential phase, did not markedly impair growth and yield (Figure 3 ).

\section{DESICCATION PROTECTION OF WHOLE CELLS}

Given the superior glass properties of hydroxyectoine, it is not surprising that (water-stressed) halophilic ectoine-type organisms have adopted a rather economical strategy to protect themselves from desiccation damage. Instead of synthesizing distinct glassforming compounds such as sucrose and/or trehalose, they simply convert, by a single enzymatic step, one compatible solute into another, hydroxyectoine, with a much higher Tg. The consequences of such a conversion from 17 to $75 \%$ hydroxyectoine (experimentally enforced by salt and temperature shock) is clearly demonstrated by a six-times increase in desiccation survivors under drastic drying conditions $\left(3 \mathrm{~h}\right.$ at $45^{\circ} \mathrm{C}$ and 10 mbar; Figure 4). This remarkable improvement was probably accomplished by the high cytoplasmic concentrations of intracellular glass-forming hydroxyectoine. Desiccation tolerance of the related C. salexigens has been investigated with cells grown under similar conditions $\left(14.5 \% \mathrm{NaCl}\right.$ and $\left.45^{\circ} \mathrm{C}\right)$ and applying the drying protocol by Manzanera et al. (2002), which entails vacuum drying at $30^{\circ} \mathrm{C}$ and $313 \mathrm{mbar}$ for $30 \mathrm{~h}$ (Reina-Bueno et al., 2012). These conditions are less drastic than those applied here, except for length of time. The authors observed a surprisingly low desiccation tolerance of approximately 5\% survivors, which was reduced even further by at least one order of magnitude in knock-out mutants unable to synthesize either hydroxyectoine or trehalose. From this they concluded that both compounds probably play a role in desiccation tolerance, although other factors must be considered. As the relative hydroxyectoine content in $C$. salexigens is similar, the much better performance of $H$. elongata is either explained as a consequence of more favorable drying conditions or of other factors triggered by temperature upshock (see below).

As we did not want to exceed the maximum growth temperature during the drying process, the temperature applied experimentally happened to be close to the $\mathrm{Tg}$ of ectoine $\left(47^{\circ} \mathrm{C}\right)$, but much below that of hydroxyectoine $\left(87^{\circ} \mathrm{C}\right)$. One would therefore assume that a slightly increased drying temperature (e.g., $50^{\circ} \mathrm{C}$ ) would enhance the difference between ectoine and hydroxyectoine as desiccation protectants.

The experimental set-up presented here enforced the cytoplasmic conversion of ectoine into hydroxyectoine, and purposely avoided the use of external excipient as an additional protective element, although one would expect a positive influence from additional external protection. Others have improved the desiccation tolerance of whole cells (both E. coli and P. putida) by external application of $1 \mathrm{M}$ trehalose or hydroxyectoine, both in combination with $1.5 \%$ polyvinyl pyrollidone (PVP) as a thickening agent (Manzanera etal., 2002, 2004a). For E. coli, they obtained survival rates of approximately $60 \%$ after vacuum drying with very little difference between trehalose and hydroxyectoine. In the case of P. putida, hydroxyectoine (approximately $40 \%$ survival) performed even better than trehalose ( $<20 \%$ survival). Hence the positive effect of hydroxyectoine as an external drying excipient (comparable to that of trehalose) appears to be met by a similarly striking effect when accumulated intracellularly. It remains to be shown whether the external addition of glass-formers (i.e., a combination of both strategies) would increase the survival rates even more (de Castro et al., 2000).

\section{OTHER POTENTIAL FACTORS INVOLVED IN DESICCATION TOLERANCE}

Inorganic ions, and in particular polyphosphates, are suspected of also playing a role in stress adaptation, either alone or in combination with other solutes (Seufferheld et al., 2008). All attempts using HPLC and ${ }^{31} \mathrm{P}-\mathrm{NMR}$ techniques to detect any major changes in inorganic cations $(\mathrm{Mg}, \mathrm{Ca}, \mathrm{Na}, \mathrm{K})$ and anions $\left(\mathrm{Cl}, \mathrm{NO}_{3}, \mathrm{SO}_{4}, \mathrm{PO}_{4}\right)$, including polyphosphates, were however, unsuccessful. It therefore appears that the conversion of ectoine into hydroxyectoine is the major observable change within low-molecular mass compounds when $H$. elongata cells prepare for a pending heat stress/desiccation event. The notable exception is potassium glutamate, the only other organic compound present in large amounts (ratio of approximately 1:4 glutamate/hydroxyectoine). Glutamate accumulation is one of the first physiological responses to salinity-induced dehydration in many microorganisms. Whether its $\mathrm{H}$-donor and $\mathrm{H}$-acceptor groups also contribute to the formation of a stabilizing H-bond network in the glassy state still needs to be clarified. These findings indicate that in particular hydroxyectoine is the crucial low-molecular mass compound for desiccation survival of $H$. elongata. It seems that the presence of at least one such glass-forming compound is essential to enable anhydrobiotic stabilization of biological systems (Tunnacliffe et al., 2001).

We cannot of course exclude that, besides hydroxylation of ectoine, other factors triggered by the combination of high salt and temperature upshock, such as heat-stress proteins, may play a role in increased desiccation protection. We were, however, unable to detect any temperature-induced proteins at the expected molecular mass. Similarly, very small IDPs $(2-30 \mathrm{kDa})$ may also play a role. Among these, late embryogenesis abundant (LEA) proteins, essential for glass formation in dormant seeds, are of particular interest (Tunnacliffe and Wise, 2007). These proteins are highly charged (due to the abundance of glutamate and lysine residues), highly hydrated and form random coils in solution. During desiccation, the formation of secondary structures releases water. Therefore, IDPs probably serve as water-retaining molecules and as scavengers for inorganic ions (ion sequestration). As such they slow down removal of water and prevent adverse effects of increased ionic strength (Tompa et al., 2006). Garay-Arroyo et al. (2000) coined the term "hydrophilins," an expanded definition of IDPs which comprises LEA proteins and dehydrins. Such proteins are characterized by a hydrophilicity index $>1$ and a glycine content 
$>6 \%$ as a distinguishing feature. In E. coli, five proteins conform to this definition (four of which respond to osmotic stress; GarayArroyo et al., 2000). One of the E. coli hydrophilins (YCIG) has been checked for its stabilization properties with $\mathrm{LDH}$ during vacuum drying, and performed well (comparable to $100 \mathrm{mM}$ trehalose) at a molar ratio of 1:1 (hydrophilin:enzyme) up to 98\% water loss (Reyes et al., 2005). A bioinformatic study on the $H$. elongata genome revealed only four uncharacterized small proteins which conform to the above criteria of hydrophilins (unpublished); however, a possible involvement in desiccation response has not yet been shown. We therefore have at present no proof that small water-binding/ ion sequestering proteins, as discussed for desiccation tolerant plants, invertebrates, cyanobacteria, fungi, and other anhydrobiotic organisms (Hand et al., 2011), may also participate in the desiccation protection of the moderately halophilic, extremely halotolerant $H$. elongata.

\section{AIR-DRYING OF MODEL ENZYME LACTATE DEHYDROGENASE AT HIGH TEMPERATURE}

The parameters of the drying process applied to model enzyme $\mathrm{LDH}$ were chosen so as to display the consequences of the vastly different Tg of the two ectoines. The drying temperature of $60^{\circ} \mathrm{C}$ lies above the $\mathrm{Tg}$ of ectoine $\left(47^{\circ} \mathrm{C}\right)$ but below that of hydroxyectoine $\left(87^{\circ} \mathrm{C}\right)$. The concentration of the enzyme $(50 \mu \mathrm{g} / \mathrm{mL})$ was chosen to minimize self-stabilizing effects (Lippert and Galinski, 1992) and excludes the interference of stabilizers from the commercial preparations. Under these conditions, hydroxyectoine performed very well (70\% residual activity, as compared to approximately $60 \%$ for sucrose and approximately $80 \%$ for trehalose) after $2 \mathrm{~h}$ drying (Figure 5). These values compare with those previously obtained after freeze-drying in $1 \mathrm{M}$ solution, all approximately 70\% (Lippert and Galinski, 1992), and those of a comparative experiment with hydrophilins and trehalose when vacuum-dried down to $2 \%$ residual water, approximately $80 \%$, as described by Reyes et al. (2005). The most striking difference here, however, lies in the fact that ectoine had no stabilizing effect whatsoever. This is very likely due to the high drying temperature, well above the $\mathrm{Tg}$ of ectoine. The fact that prolonged drying (4 and $6 \mathrm{~h}$ ) reduced residual activity, and in the case of hydroxyectoine, more severely than in the presence of sucrose and trehalose, may be explained by differences in their water-retaining capacity. As has been shown in vacuum-drying experiments of $\mathrm{LDH}$, the final water content has a great influence on residual activity, which rapidly decreases beyond $97 \%$ water loss (Reyes et al., 2005). The water-retaining capacity of hydroxyectoine has so far not been investigated, whereas the remarkable anhydrobiotic properties of trehalose have been studied in depth and accredited to its unusual polymorphism (several transitions between polymorphic crystalline and amorphous states). In particular, the dihydrate crystallites allow diffusion of water in and out of channels (Jones et al., 2006). The crystalline-glassy nanocomposite matrix of trehalose traps residual water molecules and makes it such a good material for the preservation of protein conformation (Jain and Roy, 2009; Sussich et al., 2010).

Without doubt the kinetics of water removal have a strong influence on the formation of the glassy state, and in particular the amount of retained water (Willart et al., 2002; Cesàro,
2006). This becomes also apparent when the results of the airdrying of a droplet of solute are closely investigated. Not only does this depend on the hydrophobicity of the surface, which alters microflow patterns and influences crack formation upon continued drying (Adams et al., 2008), but also on droplet size and the speed of water removal at the air-liquid interface. The evaporation of solvent creates steep solute concentrations and temperature gradients, which in turn yield aggregation of solutes, skin formation, crystallization, and most importantly, a spatial heterogeneity in the desiccated droplet (Ragoonanan and Aksan, 2008). In addition, shearing forces (resulting from crystallization effects) and mechanical stress (from cracking) may also damage biological structures in the dry state (Aksan and Toner, 2004; Jones et al., 2006). All these physical, rheological and chemical factors, when combined, tend to create an almost unpredictable outcome. As the glass-forming properties of hydroxyectoine, although suspected for a long time, have only now been proven, we still need to resolve to what extent speed of drying and residual water content influence its stabilizing effect on biomolecules and whole cells. Nevertheless, we are now able to present a novel glass-forming desiccation protectant, hydroxyectoine, which may even be able to challenge the best-known sugar-type stabilizers.

\section{ACKNOWLEDGMENTS}

This work was supported by the Deutsche Forschungsgemeinschaft (DFG) in the framework of the Graduiertenkolleg GRK 1572 "Bionics - Interactions across Boundaries to the Environment".

\section{REFERENCES}

Adams, D. R., Toner, M., and Langer, R. (2008). Microflow and crack formation patterns in drying sessile droplets of liposomes suspended in trehalose solutions. Langmuir 24, 7688-7697. doi: 10.1021/la703835w

Aksan, A., and Toner, M. (2004). Isothermal desiccation and vitrification kinetics of trehalose-dextran solutions. Langmuir 20, 5521-5529. doi: 10.1021/la0355186

Allison, S. D., Chang, B., Randolph, T. W., and Carpenter, J. F. (1999). Hydrogen bonding between sugar and protein is responsible for inhibition of dehydration-induced protein unfolding. Arch. Biochem. Biophys. 365, 289-298. doi: 10.1006/abbi.1999.1175

Arakawa, T., and Timasheff, S. N. (1985). The stabilization of proteins by osmolytes. Biophys. J. 47, 411-414. doi: 10.1016/S0006-3495(85)83932-1

Ballesteros, D., and Walters, C. (2011). Detailed characterization of mechanical properties and molecular mobility within dry seed glasses: relevance to the physiology of dry biological systems. Plant J. 68, 607-619. doi: 10.1111/j.1365313X.2011.04711.x

Belkin, S., Mehlhorn, R. J., Hideg, K., Hankovsky, O., and Packer, L. (1987). Reduction and destruction rates of nitroxide spin probes. Arch. Biochem. Biophys. 256, 232-243. doi: 10.1016/0003-9861(87)90441-3

Belton, P. S., and Gil, A. M. (1994). IR and Raman spectroscopic studies of the interaction of trehalose with hen egg white lysozyme. Biopolymers 34, 957-961. doi: 10.1002/bip.360340713

Bligh, E. G., and Dyer, W. J. (1959). A rapid method of total lipid extraction and purification. Can. J. Biochem. Physiol. 37, 911-917. doi: 10.1139/ o59-099

Bolen, D. W., and Baskakov, I. V. (2001). The osmophobic effect: natural selection of a thermodynamic force in protein folding. J. Mol. Biol. 310, 955-963. doi: 10.1006/jmbi.2001.4819

Borges, N., Ramos, A., Raven, N. D. H., Sharp, R. J., and Santos, H. (2002). Comparative study of the thermostabilizing properties of mannosylglycerate and other compatible solutes on model enzymes. Extremophiles 6, 209-216. doi: $10.1007 / \mathrm{s} 007920100236$

Buitink, J., and Leprince, O. (2004). Glass formation in plant anhydrobiotes: survival in the dry state. Cryobiology 48, 215-228. doi: 10.1016/j.cryobiol.2004. 02.011 
Bursy, J., Pierik, A. J., Pica, N., and Bremer, E. (2007). Osmotically induced synthesis of the compatible solute hydroxyectoine is mediated by an evolutionarily conserved ectoine hydroxylase. J. Biol. Chem. 282, 31147-31155. doi: 10.1074/jbc.M704023200

Cesàro, A. (2006). Carbohydrates: all dried up. Nat. Mater. 5, 593-594. doi: $10.1038 /$ nmat1701

Clegg, J. S. (2001). Cryptobiosis - a peculiar state of biological organization. Comp. Biochem. Physiol. B Biochem. Mol. Biol. 128, 613-624. doi: 10.1016/S1096-4959(01)00300-1

Coates, J. (2000). "Interpretation of infrared spectra, a practical approach," in Encyclopedia of Analytical Chemistry, ed. R. A. Meyers (Chichester: John Wiley \& Sons Ltd), 10815-10837.

Cottone, G., Ciccotti, G., and Cordone, L. (2002). Protein-trehalose-water structures in trehalose coated carboxy-myoglobin. J. Chem. Phys. 117, 9862. doi $10.1063 / 1.1518960$

Craig, I. D., Parker, R., Rigby, N. M., Cairns, P., and Ring, S. G. (2001). Maillard reaction kinetics in model preservation systems in the vicinity of the glass transition: experiment and theory. J. Agric. Food Chem. 49, 4706-4712. doi 10.1021/jf0100752

Crowe, J. H., Carpenter, J. F., and Crowe, L. M. (1998). The role of vitrification in anhydrobiosis. Annu. Rev. Physiol. 60, 73-103. doi: 10.1146/annurev.physiol.60.1.73

de Castro, A. G., Bredholt, H., Strøm, A. R., and Tunnacliffe, A. (2000). Anhydrobiotic engineering of gram-negative bacteria. Appl. Environ. Microbiol. 66 4142-4144. doi: 10.1128/AEM.66.9.4142-4144.2000

Dötsch, A., Severin, J., Alt, W., Galinski, E. A., and Kreft, J.-U. (2008). A mathematica model for growth and osmoregulation in halophilic bacteria. Microbiology 154 2956-2969. doi: 10.1099/mic.0.2007/012237-0

Dzuba, S., Golovina, Y., and Tsvetkov, Y. (1993). Echo-induced EPR spectra of spin probes as a method for identification of glassy states in biological objects. J. Magn. Reson. B 101, 134-138. doi: 10.1006/jmrb.1993.1022

Dzuba, S., Kirilina, E. P., Salnikov, E. S., and Kulik, L. V. (2005). Restricted orientational motion of nitroxides in molecular glasses: direct estimation of the motional time scale basing on the comparative study of primary and stimulated electron spin echo decays. J. Chem. Phys. 122, 94702. doi: 10.1063/1.18 56926

Francia, F., Dezi, M., Mallardi, A., Palazzo, G., Cordone, L., and Venturoli, G. (2008) Protein-matrix coupling/uncoupling in "dry" systems of photosynthetic reaction center embedded in trehalose/sucrose: the origin of trehalose peculiarity. J. Am. Chem. Soc. 130, 10240-10246. doi: 10.1021/ja801801p

Galinski, E. A., and Herzog, R. M. (1990). The role of trehalose as a substitute for nitrogen-containing compatible solutes (Ectothiorhodospira halochloris). Arch Microbiol. 153, 607-613. doi: 10.1007/BF00245273

Garay-Arroyo, A., Colmenero-Flores, J. M., Garciarrubio, A., and Covarrubias, A. A. (2000). Highly hydrophilic proteins in prokaryotes and eukaryotes are common during conditions of water deficit. J. Biol. Chem. 275, 5668-5674.doi: $10.1074 /$ jbc. 275.8 .5668

Garcia-Estepa, R., Argandoña, M., Reina-Bueno, M., Capote, N., Iglesias-Guerra, F., Nieto, J. J., et al. (2006). The ectD gene, which is involved in the synthesis of the compatible solute hydroxyectoine, is essential for thermoprotection of the halophilic bacterium Chromohalobacter salexigens. J. Bacteriol. 188, 3774-3784 doi: 10.1128/JB.00136-06

Graf, R., Anzali, S., Buenger, J., Pfluecker, F., and Driller, H. (2008). The multifunctional role of ectoine as a natural cell protectant. Clin. Dermatol. 26, 326-333. doi: 10.1016/j.clindermatol.2008.01.002

Hand, S. C., Menze, M. A., Toner, M., Boswell, L., and Moore, D. (2011). LEA proteins during water stress: not just for plants anymore. Annu. Rev. Physiol. 73 115-134. doi: 10.1146/annurev-physiol-012110-142203

Hoekstra, F. A., Golovina, E. A., and Buitink, J. (2001). Mechanisms of plant desiccation tolerance. Trends Plant Sci. 6, 431-438. doi: 10.1016/S1360-1385(01) 02052-0

Imamura, K., Sakaura, K., Ohyama, K.-I., Fukushima, A., Imanaka, H., Sakiyama T., etal. (2006). Temperature scanning FTIR analysis of hydrogen bonding states of various saccharides in amorphous matrixes below and above their glass transition temperatures. J. Phys. Chem. B 110, 15094-15099. doi: 10.1021/jp0 57527o

Jain, N. K., and Roy, I. (2009). Effect of trehalose on protein structure. Protein Sci. 18, 24-36. doi: 10.1002/pro.3
Jones, M. D., Hooton, J. C., Dawson, M. L., Ferrie, A. R., and Price, R. (2006). Dehydration of trehalose dihydrate at low relative humidity and ambient temperature. Int. J. Pharm. 313, 87-98. doi: 10.1016/j.ijpharm.2006.01.026

Kets, E. P. W., IJpelaar, P. J., Hoekstra, F. A., and Vromans, H. (2004). Citrate increases glass transition temperature of vitrified sucrose preparations. Cryobiology 48, 46-54. doi: 10.1016/j.cryobiol.2003.12.002

Knowles, P. F., Marsh, D., and Rattle, H. W. E. (1976). Magnetic Resonance of Biomolecules: An Introduction to the Theory and Practice of NMR and ESR in Biological Systems. London: John Wiley \& Sons Ltd.

Larsen, P. I., Sydnes, L. K., Landfald, B., and Strøm, A. R. (1987). Osmoregulation in Escherichia coli by accumulation of organic osmolytes: betaines, glutamic acid, and trehalose. Arch. Microbiol. 147, 1-7. doi: 10.1007/BF00492896

Lippert, K., and Galinski, E. A. (1992). Enzyme stabilization be ectoine-type compatible solutes: protection against heating, freezing and drying. Appl. Microbiol. Biotechnol. 37, 61-65. doi: 10.1007/BF00174204

Louis, P., Trüper, H. G., and Galinski, E. A. (1994). Survival of Escherichia coli during drying and storage in the presence of compatible solutes. Appl. Microbiol. Biotechnol. 41, 684-688. doi: 10.1007/BF00167285

Manzanera, M., de Castro, A. G., Tøndervik, A., Rayner-Brandes, M., Strøm, A. R., and Tunnacliffe, A. (2002). Hydroxyectoine is superior to trehalose for anhydrobiotic engineering of Pseudomonas putida KT2440. Appl. Environ. Microbiol. 68, 4328-4333. doi: 10.1128/AEM.68.9.4328-4333.2002

Manzanera, M., Vilchez, S., and Tunnacliffe, A. (2004a). High survival and stability rates of Escherichia coli dried in hydroxyectoine. FEMS Microbiol. Lett. 233, 347352. doi: 10.1016/j.femsle.2004.03.005

Manzanera, M., Vilchez, S., and Tunnacliffe, A. (2004b). Plastic encapsulation of stabilized Escherichia coli and Pseudomonas putida. Appl. Environ. Microbiol. 70, 3143-3145. doi: 10.1128/AEM.70.5.3143-3145.2004

Omayu, A., Ueno, T., and Matsumoto, A. (2008). The role of intermolecular hydrogen bonding on thermal properties of maleimide-isobutene alternating copolymers with polar groups. Macromol. Chem. Phys. 209, 1503-1514. doi: 10.1002/macp. 200800156

Potts, M. (2001). Desiccation tolerance: a simple process? Trends Microbiol. 9, 553559. doi: 10.1016/S0966-842X(01)02231-4

Ragoonanan, V., and Aksan, A. (2008). Heterogeneity in desiccated solutions: implications for biostabilization. Biophys. J. 94, 2212-2227. doi: 10.1529/biophysj.107.110684

Reina-Bueno, M., Argandoña, M., Salvador, M., Rodríguez-Moya, J., IglesiasGuerra, F., Csonka, L. N., etal. (2012). Role of trehalose in salinity and temperature tolerance in the model halophilic bacterium Chromohalobacter salexigens. PLoS ONE 7:e33587. doi: 10.1371/journal.pone.0033587

Reuter, K., Pittelkow, M., Bursy, J., Heine, A., Craan, T., Bremer, E., et al. (2010). Synthesis of 5-hydroxyectoine from ectoine: crystal structure of the non-heme iron(II) and 2-oxoglutarate-dependent dioxygenase EctD. PLoS ONE 5:e10647. doi: 10.1371/journal.pone.0010647

Reyes, J. L., Rodrigo, M.-J., Colmenero-Flores, J. M., Gil, J.-V., Garay-Arroyo, A., Campos, F., et al. (2005). Hydrophilins from distant organisms can protect enzymatic activities from water limitation effects in vitro. Plant Cell Environ. 28, 709-718. doi: 10.1111/j.1365-3040.2005.01317.x

Rodríguez-Moya, J., Argandoña, M., Iglesias-Guerra, F., Nieto, J. J., and Vargas, C. (2013). Temperature- and salinity-decoupled overproduction of hydroxyectoine by Chromohalobacter salexigens. Appl. Environ. Microbiol. 79, 1018-1023. doi: 10.1128/AEM.02774-12

Seufferheld, M. J., Alvarez, H. M., and Farias, M. E. (2008). Role of polyphosphates in microbial adaptation to extreme environments. Appl. Environ. Microbiol. 74, 5867-5874. doi: 10.1128/AEM.00501-08

Severin, J., Wohlfarth, A., and Galinski, E. A. (1992). The predominant role of recently discovered tetrahydropyrimidines for the osmoadaptation of halophilic eubacteria. J. Gen. Microbiol. 138, 1629-1638. doi: 10.1099/00221287-138-8-1629

Steiner, T. (2002). The hydrogen bond in the solid state. Angew. Chem. Int. Ed. Engl. 41, 49-76. doi: 10.1002/1521-3773(20020104)41:1<48::AID-ANIE48>3.0. $\mathrm{CO} ; 2-\mathrm{U}$

Street, T. O., Bolen, D. W., and Rose, G. D. (2006). A molecular mechanism for osmolyte-induced protein stability. Proc. Natl. Acad. Sci. U.S.A. 103, $13997-$ 14002. doi: 10.1073/pnas.0606236103

Sussich, F., Skopec, C. E., Brady, J. W., and Cesàro, A. (2010). Water mobility in the dehydration of crystalline trehalose. Food Chem. 122, 388-393. doi 10.1016/j.foodchem.2009.08.014 
Tompa, P., Bánki, P., Bokor, M., Kamasa, P., Kovács, D., Lasanda, G., et al. (2006). Protein-water and protein-buffer interactions in the aqueous solution of an intrinsically unstructured plant dehydrin: NMR intensity and DSC aspects. Biophys. J. 91, 2243-2249. doi: 10.1529/biophysj.106.084723

Tunnacliffe, A., de Castro, A. G., and Manzanera, M. (2001). Anhydrobiotic engineering of bacterial and mammalian cells: is intracellular trehalose sufficient? Cryobiology 43, 124-132. doi: 10.1006/cryo.2001.2356

Tunnacliffe, A., and Wise, M. J. (2007). The continuing conundrum of the LEA proteins. Naturwissenschaften 94, 791-812. doi: 10.1007/s00114-007-0254-y

Vanderkooi, J. M., Dashnau, J. L., and Zelent, B. (2005). Temperature excursion infrared (TEIR) spectroscopy used to study hydrogen bonding between water and biomolecules. Biochim. Biophys. Acta 1749, 214-233. doi: 10.1016/j.bbapap.2005.03.008

Vargas, C., Argandoña, M., Reina-Bueno, M., Rodríguez-Moya, J., FernandezAunion, C., and Nieto, J. J. (2008). Unravelling the adaptation responses to osmotic and temperature stress in Chromohalobacter salexigens, a bacterium with broad salinity tolerance. Saline Syst. 4, 14. doi: 10.1186/1746-1448-4-14

Wang, R., Pellerin, C., and Lebel, O. (2009). Role of hydrogen bonding in the formation of glasses by small molecules: a triazine case study. J. Mater. Chem. 19, 2747-2753. doi: 10.1039/b820294j

Widderich, N., Pittelkow, M., Höppner, A., Mulnaes, D., Buckel, W., Gohlke, H., et al. (2014). Molecular dynamics simulations and structure-guided mutagenesis provide insight into the architecture of the catalytic core of the ectoine hydroxylase. J. Mol. Biol. 426, 586-600. doi: 10.1016/j.jmb.2013.10.028

Willart, J. F., De Gusseme, A., Hemon, S., Descamps, M., Leveiller, F., and Rameau, A. (2002). Vitrification and polymorphism of trehalose induced by dehydration of trehalose dihydrate. J. Phys. Chem. B. 106, 3365-3370. doi: 10.1021/jp012836+

Wohlfarth, A., Severin, J., and Galinski, E. A. (1990). The spectrum of compatible solutes in heterotrophic halophilic eubacteria of the family Halomonadaceae. $J$. Gen. Microbiol. 136, 705-712. doi: 10.1099/00221287-136-4-705
Wolkers, W. F., Oldenhof, H., Alberda, M., and Hoekstra, F. A. (1998). A Fourier transform infrared microspectroscopy study of sugar glasses: application to anhydrobiotic higher plant cells. Biochim. Biophys. Acta 1379, 83-96. doi: 10.1016/S0304-4165(97)00085-8

Wolkers, W. F., Oliver, A. E., Tablin, F., and Crowe, J. H. (2004). A Fourier-transform infrared spectroscopy study of sugar glasses. Carbohydr. Res. 339, 1077-1085. doi: 10.1016/j.carres.2004.01.016

Zhou, Q.-H., Li, M., Yang, P., and Gu, Y. (2013). Effect of hydrogen bonds on structures and glass transition temperatures of maleimide-isobutene alternating copolymers: molecular dynamics simulation study. Macromol. Theor. Simul. 22, 107-114. doi: 10.1002/mats. 201200057

Conflict of Interest Statement: The authors declare that the research was conducted in the absence of any commercial or financial relationships that could be construed as a potential conflict of interest.

Received: 23 January 2014; paper pending published: 07 February 2014; accepted: 21 March 2014; published online: 04 April 2014.

Citation: Tanne C, Golovina EA, Hoekstra FA, Meffert A and Galinski EA (2014) Glassforming property of hydroxyectoine is the cause of its superior function as a desiccation protectant. Front. Microbiol. 5:150. doi: 10.3389/fmicb.2014.00150

This article was submitted to Extreme Microbiology, a section of the journal Frontiers in Microbiology.

Copyright (c) 2014 Tanne, Golovina, Hoekstra, Meffert and Galinski. This is an openaccess article distributed under the terms of the Creative Commons Attribution License (CC BY). The use, distribution or reproduction in other forums is permitted, provided the original author(s) or licensor are credited and that the original publication in this journal is cited, in accordance with accepted academic practice. No use, distribution or reproduction is permitted which does not comply with these terms. 